\title{
ARTICLE
}

\section{CZ-7, a new derivative of Claulansine F, ameliorates 2VO-induced vascular dementia in rats through a Nrf2-mediated antioxidant responses}

\author{
Dan-dan Liu ${ }^{1}$, Xia Yuan ${ }^{2}$, Shi-feng Chu ${ }^{1}$, Chen Chen ${ }^{1}$, Qian Ren ${ }^{1}$, Piao Luo ${ }^{3}$, Mei-yu Lin ${ }^{3}$, Sha-sha Wang ${ }^{1}$, Tian-bi Zhu ${ }^{3}$, Qi-di Ai ${ }^{3}$, \\ Ying-da Zang ${ }^{1}$, Dong-ming Zhang ${ }^{1}$, Xin He ${ }^{4}$, Zhi-hua Huang ${ }^{5}$, Hong-shuo Sun ${ }^{5}$, Zhong-ping Feng ${ }^{5}$ and Nai-hong Chen ${ }^{1,3}$
}

Vascular dementia (VD) results from accumulated damage in the vascular system, which is characterized by progressive impairments in memory and cognition and is second only to Alzheimer's disease (AD) in prevalence among all types of dementia. In contrast to $A D$, there is no FDA-approved treatment for VD owing to its multiple etiologies. In this study, we investigated whether CZ-7, a new derivative of Claulansine F (Clau F) with verified neuroprotective activity in vitro, could ameliorate the cognitive impairment of rats with permanent occlusion of bilateral common carotid arteries (2VO) and its potential mechanisms of action. The $2 \mathrm{VO}$ rats were orally administered CZ-7 $(10,20,40 \mathrm{mg} / \mathrm{kg})$ from day 27 to day 53 post-surgery. Morris water maze tests conducted at day $48-51$ revealed that CZ-7 administration significantly reduced the escape latency in $2 \mathrm{VO}$ rats. After the rats were sacrificed on day 53, morphological studies using Nissl and terminal deoxynucleotidyl transferase dUTP nick end labeling (TUNEL) staining showed that administration of CZ-7 markedly attenuated the pathological changes in CA1-CA3 area of the hippocampus, including neuronal cell loss, nuclear shrinkage, and dark staining of neurons, and significantly decreased the chronic cerebral hypoperfusion-induced cell loss. Klüver-Barrera staining study revealed that CZ-7 administration significantly improved the white matter lesions. 8-OHdG and reactive oxygen species (ROS) immunofluorescent analyses showed that CZ-7 administration significantly decreased oxidative stress in CA1-CA3 area of the hippocampus. Finally, we found that the CZ-7-improved oxidative stress might be mediated via the Nrf2 pathway, evidenced by the double immunofluorescent staining of Nrf2 and the elevation of expression levels of oxidative stress proteins HO-1 and NQO1. In conclusion, CZ-7 has therapeutic potential for VD by alleviating oxidative stress injury through Nrf2-mediated antioxidant responses.

Keywords: claulansine F; CZ-7; vascular dementia; 2VO rats; chronic cerebral hypoperfusion; spatial memory; oxidative stress; Nrf2; HO-1; NQO1

Acta Pharmacologica Sinica (2019) 40:425-440; https://doi.org/10.1038/s41401-018-0078-7

\section{INTRODUCTION}

The dramatic increase in the number of elderly people throughout the world has brought attention to aging-related degenerative diseases, such as dementia. Dementia has catapulted into the public's consciousness in the past few years [1]. Vascular dementia (VD) often results from accumulated damage to the vascular system. VD is characterized by progressive impairments in memory and cognition and is second only to Alzheimer's disease (AD) in prevalence among all types of dementia [2]. Various mechanisms interact with each other and lead to the complex pathophysiological processes of VD. VD is mostly attributed to stroke or deep white matter lesions detected on imaging, and stroke risk factors are independently associated with an increased risk of vascular cognitive impairment [3]. In contrast to $A D$, there is no FDA-approved treatment for VD owing to its multiple etiologies [4]. At present, several drugs employed for VD are conventionally used for $A D$ or are known to lower cardiovascular risk factors [5], such as cholinesterase inhibitors. Cholinesterase inhibitors might be appropriate treatments for VD because they improve cognitive function, clinical global impression, and activities of daily life in living patients with probable or possible mild to moderate VD [6]. In general, the current therapies aim to merely improve symptomatology or are of uncertain clinical significance [7]. In the coming decades, there will be a marked increase in the number of cognitively impaired stroke survivors, especially among elderly people [8]. Therefore, it is essential to develop more effective and targeted anti-VD drugs.

Clausenamide (Clau) is a newly found alkaloid that is isolated from the leaves of Clausena lansium (Lour) Skeels, and an evaluation of its activity showed that the principal pharmacological effects of (-)-Clau

\footnotetext{
${ }^{1}$ State Key Laboratory of Bioactive Substances and Functions of Natural Medicines, Institute of Materia Medica \& Neuroscience Center, Chinese Academy of Medical Sciences and Peking Union Medical College, Beijing 100050, China; ${ }^{2}$ State Key Laboratory of Natural and Biomimetic Drugs, School of Pharmaceutical Sciences, Peking University, Beijing 100191, China; ${ }^{3}$ Hunan University of Chinese Medicine, Changsha 410208, China; ${ }^{4}$ Tianjin University of Traditional Chinese Medicine, Tianjin 300193 , China and ${ }^{5}$ University of Toronto, Toronto, ON M5S 1A8, Canada

Correspondence: Nai-hong Chen (chennh@imm.ac.cn)

These authors contributed equally: Dan-dan Liu, Xia Yuan.
}

Received: 7 November 2017 Accepted: 20 May 2018

Published online: 31 October 2018 
were to increase the cognition of mice/rats and inhibit, to different degrees, the pathological changes of $A D$ through its multi-target effects $[9,10]$. Claulansine $F$ (Clau F) is a new carbazole alkaloid isolated from the stem of $C$. lansium (Lour) Skeels [11]. Our research group conducted a series of studies on Clau $F$ and found that (1) Clau $\mathrm{F}$ suppressed apoptosis and oxidative stress induced by sodium nitroprusside in PC12 cells [12]; (2) Clau F promoted neuritogenesis in PC12 cells via the ERK signaling pathway [13]; and (3) Clau F promoted neuronal differentiation of neural stem and progenitor cells through the Akt/GSK-3 $\beta / \beta$-catenin pathway [14]. Based on the structure of Clau F, 37 new derivatives (named compounds CZ1-37) were obtained by Dong-Ming Zhang's research group to seek more effective and safer compounds. Preliminary studies on the activity of Clau $\mathrm{F}$ and its derivatives showed that the activities of some derivatives were better than that of Clau F. Among them, derivative CZ-7 exhibited the strongest neuroprotective effects in vitro and inhibited programmed death of PC12 cells and primary cortical neurons [15]. Preliminary metabolic studies showed that CZ-7 readily passed through the blood-brain barrier and that its exposure in the brain was 4.3 times that of the plasma. In addition, CZ-7 showed strong free radical-scavenging activity [15]. Preliminary acute toxicological test results showed that $\mathrm{CZ}-7$ had no toxic side effects with an oral $1 \mathrm{~g} / \mathrm{kg}$ dose in ICR mice. In the light of the above findings, we designed this study to investigate the utility of CZ-7 in chronic cerebral hypoperfusion-induced VD in rats and its potential mechanism of action.

\section{MATERIALS AND METHODS}

Drugs and reagents

CZ-7 (purity $>98 \%$ by HPLC analysis) was synthesized by DongMing Zhang's research group of the Institute of Materia Medica, Chinese Academy of Medical Sciences. The chemical structures of Clau F and CZ-7 are shown in Fig. 1. For oral administration, CZ-7 was first dissolved in dimethylsulfoxide (DMSO) and then $0.5 \%$ CMC-Na solution (e.g., CZ-7:DMSO:5\% CMC-Na $=400 \mathrm{mg}: 1$ $\mathrm{mL}: 100 \mathrm{~mL}$ ). Nimodipine was purchased from Bayer AG (Leverkusen, Germany) and dissolved in $0.5 \%$ CMC-Na solution. Total proteins were quantified by Beyotime Biotechnology (Beijing, China). Nuclear and cytoplasmic proteins from hippocampus were extracted by Nuclear-Cytosol Extraction Kit (Applygen Technologies, Inc., Beijing, China). The antibodies used for Western blotting and immunofluorescence are shown in Table 1. All other reagents were of analytical grade and were purchased locally.

\section{Animals}

Male Wistar rats $(260 \pm 10 \mathrm{~g}$, supplied by Vital River Laboratories, Beijing, China) were housed in groups of four per cage at a temperature of $23 \pm 1{ }^{\circ} \mathrm{C}$, on a $12 \mathrm{~h}$ light-dark cycle, and food and water were provided ad libitum. All experiments were performed in accordance with the guidelines established by the National Institutes of Health Guide for the Care and Use of Laboratory Animals and were approved by the Animal Care Committee of the Peking Union Medical College and Chinese Academy of Medical Sciences (Beijing, China).

Surgical procedure

Permanent occlusion of bilateral common carotid arteries (2VO) was utilized to cause a permanent and persistent cerebral

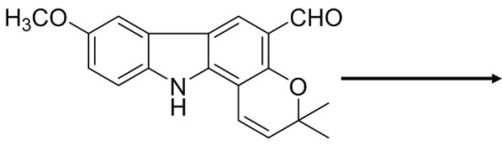

Clau F

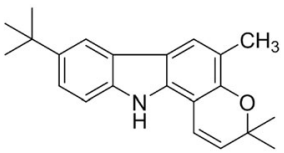

$\mathrm{CZ}-7$
Fig. 1 Chemical structures of Clau F and CZ-7

\begin{tabular}{|c|c|c|c|}
\hline Target & Host & $\begin{array}{l}\text { Dilution } \\
\text { (usage) }\end{array}$ & Supplier \\
\hline \multirow[t]{2}{*}{ Nrf2 } & Rat monoclonal & 1:200 (WB) & Santa Cruz Biotechnology \\
\hline & $\begin{array}{l}\text { Mouse } \\
\text { monoclonal }\end{array}$ & $1: 200$ (IF) & Abcam, United States \\
\hline HO-1 & Rat monoclonal & $1: 500$ (WB) & $\begin{array}{l}\text { Proteintech Group, } \\
\text { Chicago, USA }\end{array}$ \\
\hline NQO1 & Rat monoclonal & 1:500 (WB) & $\begin{array}{l}\text { Proteintech Group, } \\
\text { Chicago, USA }\end{array}$ \\
\hline$\beta$-actin & $\begin{array}{l}\text { Mouse } \\
\text { monoclonal }\end{array}$ & 1:5000 (WB) & Sigma, St Louis, MO, USA \\
\hline lba-1 & Rat monoclonal & $1: 250$ (IF) & Wako, Japan \\
\hline GFAP & Rat monoclonal & $1: 200$ (IF) & Dako, Japan \\
\hline MAP2 & Rat monoclonal & $1: 200$ (IF) & Abcam, United States \\
\hline PCNA & Rat monoclonal & 1:500 (WB) & Santa Cruz Biotechnology \\
\hline 8-OHdG & $\begin{array}{l}\text { Mouse } \\
\text { monoclonal }\end{array}$ & $1: 500$ (IF) & Sigma, St Louis, MO, USA \\
\hline
\end{tabular}

hypoperfusion in rats [16]. All rats had access to water but not food for the $12 \mathrm{~h}$ before $2 \mathrm{VO}$ surgery. Each rat was anesthetized with a mixture of $5 \%$ isoflurane and $95 \%$ oxygen, and then a mixture of $3 \%$ isoflurane and $97 \%$ oxygen was supplied by a respiratory mask during the surgery. In the permanent $2 \mathrm{VO}$ group, through a $1.5-2 \mathrm{~cm}$ midline cervical incision, both common carotid arteries were carefully exposed and doubly ligated with 5-0 silk sutures just below the bifurcation into internal and external carotid arteries and then cauterized from the middle of the two silk sutures. The sham-operated animals were treated similarly to the $2 \mathrm{VO}$ rats without carotid artery ligations and without cauterization. Then, we sprinkled an appropriate amount of penicillin powder into the wound and closed it carefully.

Drug administration and experimental design

The Morris water maze (MWM) test was used in two stages in the behavioral experiment. The first stage was carried out on days 22-26 after operation to evaluate the cognitive deficit of each ischemic rat. We rejected the unsuccessful rats. The mean time required to reach the hidden platform of each rat during the acquisition stage on five consecutive days was calculated. The mean time of each $2 \mathrm{VO}$ rat was defined as value 1 , and the mean time of all rats in the sham group was value 2 . The screening criterion (SC) was selected as the index for evaluating the cognitive deficit of each ischemic rat: $\mathrm{SC}=$ (value 1 -value 2 )/ value 1. A rat was considered to have a cognitive deficit if its SC was greater than 0.2 [17]. The sham-operated rats treated with $0.5 \%$ CMC-Na served as the sham group. $2 \mathrm{VO}$ rats with a ratio larger than 0.2 were randomly divided into five groups: (1) $2 \mathrm{VO}$ rats treated with $0.5 \%$ CMC-Na (model group); (2) $2 \mathrm{VO}$ rats treated with CZ-7 $10 \mathrm{mg} / \mathrm{kg}$; (3) $2 \mathrm{VO}$ rats treated with CZ-7 $20 \mathrm{mg} / \mathrm{kg}$; (4) $2 \mathrm{VO}$ rats treated with CZ-7 $40 \mathrm{mg} / \mathrm{kg}$; (5) $2 \mathrm{VO}$ rats treated with nimodipine $20 \mathrm{mg} / \mathrm{kg}$. Every group consisted of 10 rats with identical mean body weights. Daily oral administration of CZ-7 (10, $20,40 \mathrm{mg} / \mathrm{kg})$, nimodipine $(20 \mathrm{mg} / \mathrm{kg})$, or vehicle $(0.5 \% \mathrm{CMC}-\mathrm{Na})$ started on day 27 post-surgery and lasting until the termination of the experiment on day 53. The second-stage MWM test was carried out on days 48-52 after operation to evaluate the effect of CZ-7 on improvement of cognitive deficits. Behavioral tests were conducted $4 \mathrm{~h}$ after drug administration. The timeline for surgical procedures (2VO), drug treatment, and behavioral tests was shown in Fig. 2. 


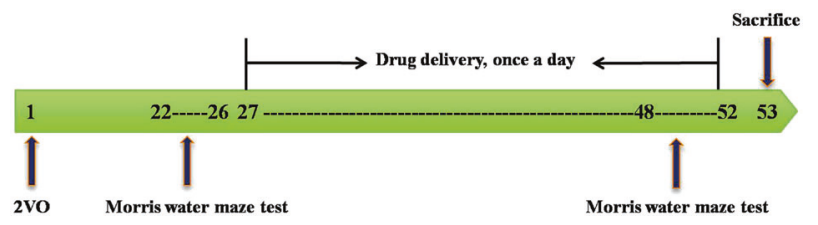

Fig. 2 Timeline for surgical procedures (2VO), drug treatment, and behavioral tests

\section{MWM test}

The MWM test was performed as previously described [18]. The apparatus consisted of a circular water tank $120 \mathrm{~cm}$ in diameter and $50 \mathrm{~cm}$ in height, filled to a depth of $30 \mathrm{~cm}$ with water at $23 \pm$ $1{ }^{\circ} \mathrm{C}$ to cover a black platform $(10 \mathrm{~cm}$ in diameter). The tank was divided into four quadrants called I, II, III, and IV at equal distances from the rim. The platform was located in the center of quadrant I during training. The top of the platform was approximately $1.5 \mathrm{~cm}$ below the surface of the water. During the training period of the task, rats were given two trials (in quadrants III and IV, respectively) per day at an interval of $10 \mathrm{~s}$ to find the hidden platform on five consecutive days. The experimenter conducting the MWM test was blinded to the treatment groups (double-blind). The rat was gently placed into the water, facing the side walls of the maze from one of the four starting positions. The swimming path of the rat was monitored by a video camera linked to a computer through an image analyzer (Chinese Academy of Medical Sciences, Beijing, China). For each training trial, the latency to escape onto the hidden platform and the path length were recorded. The rat was given a maximum of $60 \mathrm{~s}$ to find the hidden platform. If the rat failed to find the platform within $60 \mathrm{~s}$, the training was terminated and a maximum score of $60 \mathrm{~s}$ was assigned. The rat was then guided to the hidden platform, and it was allowed $10 \mathrm{~s}$ reinforcement on the platform. In the second stage of the MWM test, after the last training trial on training day 4 , a probe test, in which the hidden platform was removed, was conducted the next day. The rat was allowed to swim freely for 60 $s$ in the pool before it was removed from water, and the number of times it crossed the platform and the mean time spent in the target quadrant were recorded as an assessment of spatial memory.

\section{Neuropathological analysis}

Three rats chosen randomly from each group were anesthetized with chloral hydrate $(350 \mathrm{mg} / \mathrm{kg}$, ip) at day 53 and then perfused transcardially with phosphate-buffered saline (PBS) followed by $4 \%$ paraformaldehyde. The whole brain was removed and then post-fixed in the same paraformaldehyde. It was gradually dehydrated by sucrose polyformaldehyde and subsequently embedded in paraffin blocks. Then, $4-\mu m$-thick coronal sections were prepared for neuropathological analysis and immunofluorescence staining.

For Nissl staining, hippocampus paraffin coronal tissues were deparaffinized and hydrated. The sections were immersed in $0.2 \%$ cresyl violet for $5 \mathrm{~min}$ and then covered with xylene-based mounting medium. Nissl-stained cells in the hippocampal CA1-CA3 area were observed at $\times 200$ magnifications. For TUNEL staining to identify apoptotic cells, according to the manufacturer's instructions, paraffin-embedded sections were pre-treated with proteinase $\mathrm{K}(20 \mu \mathrm{g} / \mathrm{mL})$ in Tris-hydrochloride buffer for 30 $\min$ at $37^{\circ} \mathrm{C}$. After washing three times with PBS, the sections were incubated in TUNEL reaction mixture for $1 \mathrm{~h}$ at $37^{\circ} \mathrm{C}$ in the dark. After washing three times with PBS, nuclear staining was identified in cell nuclei with Hoechst (1:1000). TUNEL-positive cells in hippocampal CA1-CA3 region were observed at $\times 200$ magnification. For Klüver-Barrera staining, coronal hippocampus paraffin sections were deparaffinized and hydrated, then stained with Klüver-Barrera Luxol fast blue. The severity of the white matter lesions was graded into four ranks by an independent investigator blinded to the treatment group: normal (grade 0), disarrangement of the nerve fibers (grade 1), the formation of marked vacuoles (grade 2), and the disappearance of myelinated fibers (grade 3) [19]. Representative photographs of tissue sections stained with Klüver-Barrera in the corpus callosum (cc), external callosum (ec), and internal capsule (ic) of the white matter were taken at $\times 200$ magnifications. Sections of Nissl staining and Klüver-Barrera staining were observed using a light microscope, and TUNEL staining was observed using a laser scanning confocal microscope (Leica TCSSP2, Solms, Germany).

\section{Immunofluorescence staining}

For immunofluorescence staining, after dewaxing and dehydration, hippocampus paraffin tissues were incubated for antigen retrieval for $10 \mathrm{~min}$. Then, the slices were placed at room temperature and washed three times for 5 min with PBS. Next they were soaked in $0.5 \%$ Triton X-100 for $10 \mathrm{~min}$ and washed three times for $5 \mathrm{~min}$ with PBS, followed by blockade with goat serum and incubation with a primary antibody against Nrf2, MAP2, GFAP, mouse anti-8-hydroxy-2'deoxyguanosine (8-OHdG), or Iba-1 at $4{ }^{\circ} \mathrm{C}$ overnight. Subsequently, sections were washed with PBST three times and incubated for $2 \mathrm{~h}$ at room temperature in the dark with secondary fluorescence antibodies. All images were captured under a laser scanning confocal microscope (Leica TCSSP2, Solms, Germany). The entire quantifying procedure was performed in a blinded manner.

ROS production measurement by dihydroethidium staining Brain sections $(4 \mu \mathrm{m})$, after dewaxing and dehydration, were incubated for antigen retrieval for $10 \mathrm{~min}$, then incubated with dihydroethidium (100 $\mu \mathrm{M}$; Molecular Probes, Sigma, D7008) in the dark for $3 \mathrm{~h}$ at $37^{\circ} \mathrm{C}$, followed by three washes with PBST. Next, the sections were incubated with Hoechst $(1: 1000)$ for 15 min at $37^{\circ} \mathrm{C}$. The slides were rinsed three times with PBST, and mounted with $90 \%$ glycerin and coverslipped. The tissue sections were then visualized with a confocal laser scanning microscope, and fluorescence was detected.

Western blotting analysis

On day 53 , the bilateral hippocampal tissues were excised at $4{ }^{\circ} \mathrm{C}$ and stored at $-80^{\circ} \mathrm{C}$ until use. The cytoplasm and nuclear proteins were extracted from the hippocampus using a nuclear-cytosol extraction kit (Applygen, Beijing, China) according to its manufacturer's instructions. The hippocampus tissues were homogenized and dissociated with lysis buffer (in $150 \mathrm{mM} \mathrm{NaCl}, 25 \mathrm{mM}$ Tris-HCl, 1 mM EGTA, 1 mM EDTA, pH 7.4, 1\% Triton X-100, 1 mM PMSF) for $30 \mathrm{~min}$ and then centrifuged at $12000 \times g$ for $30 \mathrm{~min}$; the supernatants were collected after centrifugation and used for Western blotting analysis. Protein concentrations were determined using the BCA protein assay. Next, samples were separated by sodium dodecyl sulfate-polyacrylamide gel electrophoresis and transferred onto PVDF membranes. The membranes were blocked with $3 \%$ bovine serum albumin in Tris-buffered saline for $2 \mathrm{~h}$. Then, the membranes were incubated at $4{ }^{\circ} \mathrm{C}$ overnight with the Nrf2, NQO1, HO-1, PCNA, or $\beta$-actin antibody. After washing three times with TBST the next day, the membranes were incubated with a horseradish peroxidase-conjugated secondary antibody (goat anti-rabbit, 1:5000; goat anti-mouse, 1:5000) (Santa Cruz Biotechnologies, Santa Cruz, CA) for $2 \mathrm{~h}$ at room temperature and processed for visualization by enzyme-linked chemiluminescence. The bands were detected using the Enhanced Chemiluminescence Plus detection system (GE Healthcare, Fairfield, CT, USA). The density of each band was quantified using Quantity One software (Tokyo, Japan). The data are expressed as ratios and were normalized to the amount of $\beta$-actin or PCNA ( $n=4$ for each group). 
428

Image analysis

The number of Nissl bodies and staining for TUNEL, Klüver-Barrera, 8-OHdG, and ROS were assessed in full microscopic fields $(\times 200)$ of the hippocampus or white matter by using the digital medical image analysis software Image-Pro Plus (Media Cybernetics, Silver Spring, MA, USA). Three random nonoverlapping sections were viewed and counted under a grid at $\times 200$ magnification.

Statistical analysis

Data are presented as the mean \pm SEM. Raw data were statistically analyzed with Graph Pad Prism 5.0. MWM test escape latencies and swimming speeds were analyzed using two-way analysis of variance (ANOVA) with repeated measures. The other data were analyzed using one-way ANOVA. Fisher's least-significantdifference post hoc test was used to test the differences between two groups. A value of $P<0.05$ was considered statistically significant.

\section{RESULTS}

CZ-7 attenuated spatial cognitive impairment induced by chronic cerebral hypoperfusion

Learning and memory retention of the MWM test was used to evaluate spatial memory in rats. As shown in Fig. 3a, b, the escape latency (time to find the hidden platform) declined progressively during days $22-26$ in all rats. However, the $2 \mathrm{VO}$ rats had longer escape latencies throughout the training days than shamoperated rats $(P<0.05$ or $P<0.001)$, demonstrating that hypoperfusion successfully induced learning deficit in this rat $2 \mathrm{VO}$ model. As presented in Fig. 3c, compared with the sham-operated group, the body weight of the $2 \mathrm{VO}$ group began to recover gradually on the fifth day after the operation. After CZ-7 treatment (10, 20, 40 $\mathrm{mg} / \mathrm{kg}$ ) for approximately 4 weeks, the escape latency was significantly shortened compared with CMC-Na-treated 2VO group on days 2 and 4 (Fig. $3 \mathrm{~d}$ ). In the probe trial, the number of platform crossings and the mean time spent in the target quadrant were used to evaluate the retention performance. The CMC-Na-treated $2 \mathrm{VO}$ rats had fewer crossings and less mean time spent in the target quadrant than other groups (Fig. 3e, f). CZ-7 treatment at the dose of $20 \mathrm{mg} / \mathrm{kg}$ improved those deficits, but not significantly. The groups treated with $\mathrm{CZ}-7$ at the doses of 10 and $40 \mathrm{mg} / \mathrm{kg}$ and with nimodipine showed marginal improvements in learning the task. Although there was no significant difference between the CZ-7 and CMC-Na treatment groups, the effect of CMC-Na was inferior to that of CZ-7 or nimodipine. To determine whether the group differences in escape latency were due to difference in swimming ability, swimming speed (path length/escape latency) was calculated for each group. As shown in Fig. $3 g$, there was no difference between the groups in swimming ability.

The effects of CZ-7 on cerebral neuropathological alterations induced by chronic cerebral hypoperfusion

The hippocampus is considered one of the most important brain regions associated with learning and memory and is particularly susceptible to ischemic insult. Neuronal death in the hippocampus is a major contributor to memory decline [20]. Representative microphotographs of Nissl staining in the hippocampus 53 days after $2 \mathrm{VO}$ are shown in Fig. 4. Extensive neuronal changes were visualized in the CA1-CA3 area of the hippocampus: neuronal cell loss, nuclear shrinkage, and dark staining of neurons. Long-term administration of CZ-7 or nimodipine markedly reduced these pathological changes and attenuated the chronic cerebral hypoperfusion-induced cell loss in CA1-CA3. Compared with the pronounced damage in CA1-CA3, chronic cerebral hypoperfusion did not induce obvious neuronal loss in the dentate gyrus (data not shown). TUNEL staining is commonly used in the detection of neurons undergoing apoptosis. As reflected in the Fig. 5, 2VO rats treated with CMC-Na exhibited more TUNELpositive neurons in the CA1-CA3 area. The nuclei of the model group's CA1-CA3 region were irregularly shaped and degenerating. The $2 \mathrm{VO}$ group treated with CZ-7 at $20 \mathrm{mg} / \mathrm{kg}$ had markedly fewer TUNEL-positive cells, suggesting that CZ-7 protected neurons from apoptosis.

Chronic cerebral hypoperfusion is an important cause of white matter injury. The pathology of the $2 \mathrm{VO}$ model is attributed not only to hippocampus damage but also to white matter disintegration [21]. As illustrated in Fig. 6, myelinated fibers in the corpus callosum (cc), external callosum (ec), and internal capsule (ic) of the white matter were stained with Klüver-Barrera, and no obvious cell damage was observed in the sham-operated rats. In contrast, CMC-Na-treated $2 \mathrm{VO}$ rats showed severe rarefaction, marked vacuolization, increased crookedness, and disarrangement of myelin fibers in the $\mathrm{cc}$, ec, and ic. Administration of CZ-7 or nimodipine attenuated these lesions in white matter, especially the CZ-7 $20 \mathrm{mg} / \mathrm{kg}$ group.

\section{CZ-7 suppressed chronic cerebral hypoperfusion-induced} oxidative stress in hippocampus

DNA damage is usually repaired by a specific system and produces various oxidized metabolites. One of the recognized biomarkers of oxidative damage of DNA is 8-OHdG [22]. Immunofluorescence for $8-\mathrm{OHdG}$ in the hippocampus of rats was performed to measure DNA-oxidative damage after chronic cerebral hypoperfusion [23] with or without CZ-7. As shown in Fig. 7, the 8-OHdG-positive cells increased significantly in the model group. The $8-\mathrm{OHdG}$-positive cells of the CA1 and CA3 areas of the hippocampus in the CZ-7treated groups were obviously decreased compared with the model group. These results show that CZ-7 prevented oxidative DNA damage in rats.

\section{CZ-7 rescued ROS impairment and protected neurons in} hippocampus of chronic cerebral hypoperfusion rats

The most important free radicals in the human body are ROS, which are by-products of cellular metabolism that are largely generated from mitochondria [24]. ROS are involved in the maintenance of redox homeostasis and various cellular signaling pathways, but excessive accumulation of ROS can cause oxidative damage to DNA, resulting in the production of 8 -OHdG [25]. On the basis of the above results, we examined the ROS using immunofluorescence analysis. As shown in Fig. 8, chronic cerebral hypoperfusion induced robust production of ROS in the rat hippocampus $C A 1$ and $C A 3$ regions, which were scavenged in the groups treated with $C Z-7(10,20$, and $40 \mathrm{mg} / \mathrm{kg})$. These results further suggest that CZ-7 might have antioxidant properties.

CZ-7 induced Nrf2 translocation from the cytoplasm to the nucleus in hippocampus of chronic cerebral hypoperfusion rats Considering Nrf2 is a master regulator of cytoprotective regulators against oxidative stress, we hypothesized that the ameliorated cognitive deficits and ischemic injury after CZ-7 treatment in 2VO rats might be related to an increase in Nrf2 activation. We investigated whether ROS triggered the members of the Nrf2 pathway and its downstream products. We examined the activation of Nrf2 using double-immunofluorescence staining to identify the nuclear translocation of Nrf2 in neurons after $2 \mathrm{VO}$ surgery and the source of Nrf2. As shown in Fig. 9a-f, staining for Nrf2 with Iba-1, GFAP, or MAP2 showed that $2 \mathrm{VO}$ induced Nrf2 chiefly in neurons rather than in astrocytes or microglia in the hippocampus of rats. As shown in Fig. 9e, f, the Nrf2 was mainly located in the cytoplasm in the model group, whereas the CZ-7 treatment induced Nrf2 translocation from the cytoplasm to the nucleus. In the model group, the neurons were markedly shrunken and pyknotic, the number of cells decreased, and some cells also 
a

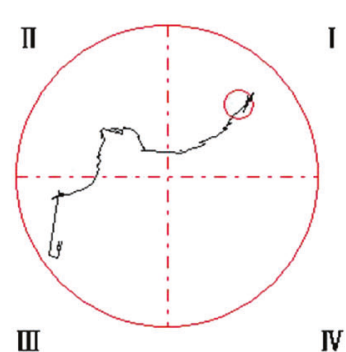

IV b

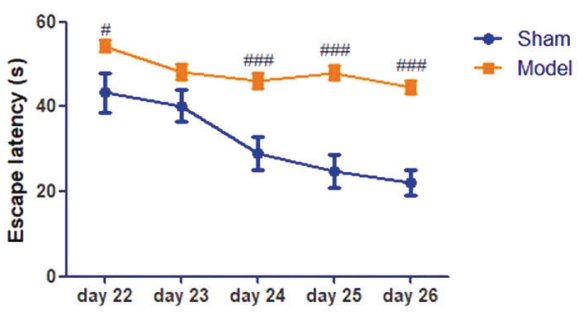

Sham
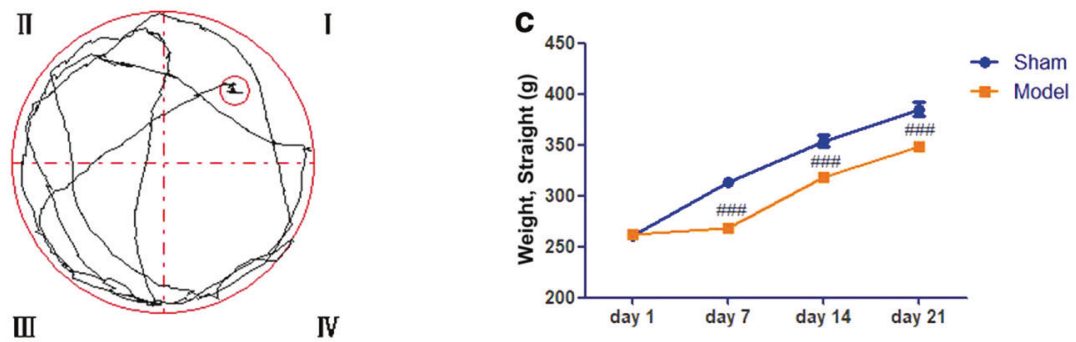

Model

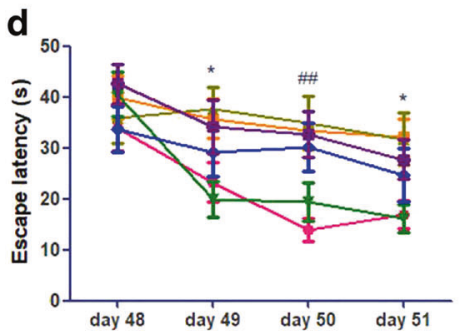

- Sham

-- Model

- CZ-7-10 mg/kg

$\rightarrow \mathrm{CZ}-7-20 \mathrm{mg} / \mathrm{kg}$

- CZ-7-40 mg/kg

- Nimodipine
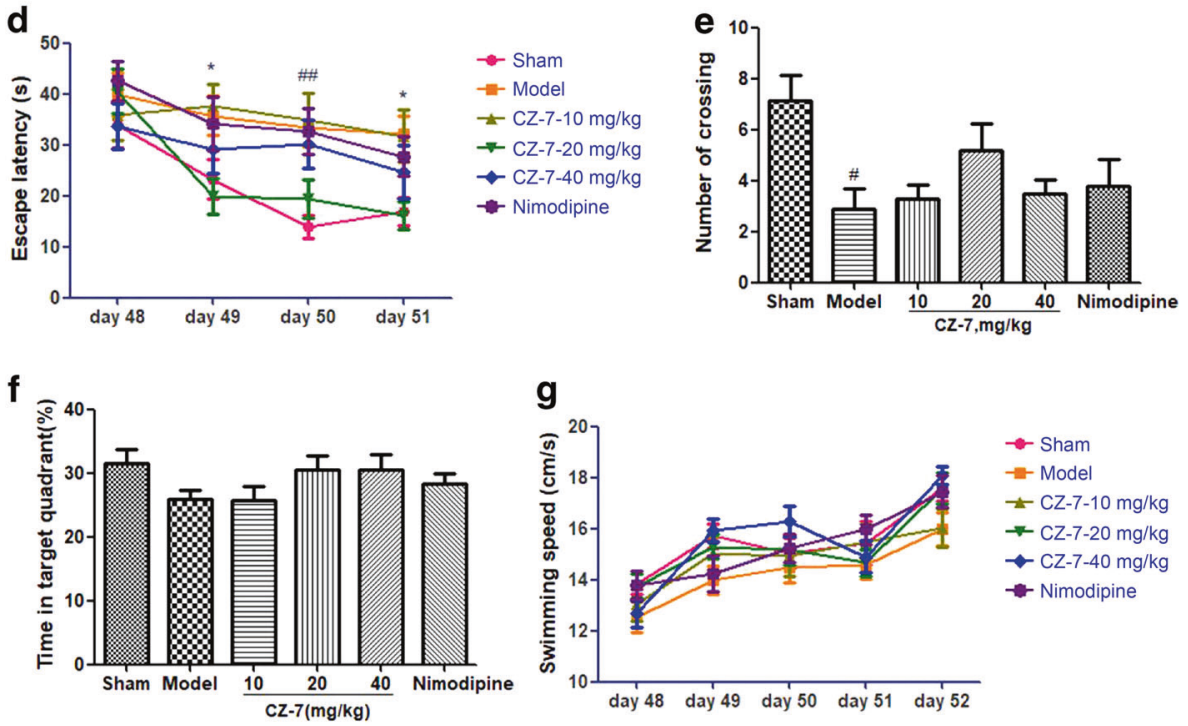

Fig. 3 a The performance in the Morris water maze test (days 22-26 post-2VO) in sham rats and in rats with memory deficit induced by permanent occlusion of the bilateral common carotid arteries. b Escape latency from the start point to the hidden platform at days 22-26, before

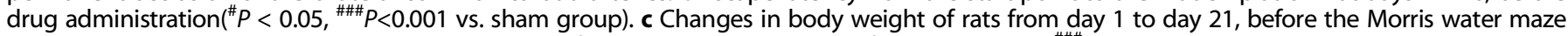
test. Values are expressed as the mean $\pm \operatorname{SEM}(n=10$ for sham group and $n=70$ for model group, \#\#\# $P<0.001$ vs. sham group). For Fig. b, two-way ANOVA and post hoc Newman-Keuls test for escape latency: 2VO surgery, $F(1,305)=82.19, P<0.0001 ;$ time, $F(4,305)=9.68, P<0.0001$; interaction, $F(4,305)=2.80, P=0.0263$. For Fig. $c$, two-way ANOVA and post hoc Newman-Keuls test for weight: $2 \mathrm{VO}$ surgery, $F(1,244)=56.93$, $P<0.0001$; time, $F(3,244)=143.83, P<0.0001$; interaction, $F(3,244)=6.68, P=0.0002)$. $\mathbf{d}$ Time to locate the hidden platform of each group at days $48-51\left({ }^{\# \#} P<0.01\right.$ vs. sham group; ${ }^{*} P<0.05$ vs. model group). e Number of crossings in the target quadrant within $60 \mathrm{~s}$ in the probe trial (no platform) at day 52 ( $P<0.05$ vs. sham group). f Percentage of time spent in the target quadrant within $60 \mathrm{~s}$ in the probe trial (no platform) at day 52. g Swimming speed (path length/escape latency) was not different between the groups. Values are expressed as the mean \pm SEM $(n=10)$. For Fig. d, two-way ANOVA and post hoc Newman-Keuls test for escape latency: drug, $F(4,340)=5.06, P=0.0006 ;$ time, $F(3,340)=6.79, P=$ 0.0002 ; interaction, $F(12,340)=0.87, P=0.5750$. For Fig. e, one-way ANOVA, $F(5,46)=3.230, P=0.0150$. For Fig. $\mathbf{f}$, one-way ANOVA, $F(5,52)=$ $1.568, P=0.1875$. For Fig. g, two-way ANOVA and post hoc Newman-Keuls test for swimming speed: drug, $F(4,390)=2.92, P=0.0211 ;$ time, $F(4$, $390)=23.13, P<0.0001$; interaction, $F(16,390)=0.91, P=0.5609)$ 
430

displayed an irregular shape and a tangled appearance. In contrast, in the CZ-7-treated groups, the nucleus showed normal morphological features, and the number of cells in the hippocampus decreased only sporadically. These results are consistent with the Nissl and TUNEL staining (Figs. 4 and 5).

Effects of CZ-7 on the expression of oxidative proteins in hippocampus of chronic cerebral hypoperfusion rats

We then measured the Nrf2 protein expression in nuclear extracts, cytoplasmic extracts, and total protein extracts of hippocampus using Western blotting in all the groups. As reflected in Fig. 10a, b, compared with the sham group, the model group had an obvious decrease in the ratio of nuclear to cytoplasmic Nrf2 and an increase in total Nrf2. Compared with the model group, CZ-7 treatment resulted in a pronounced enhancement of nuclear and total Nrf2 expression. HO-1 and NQO1 are crucial downstream proteins regulated by Nrf2. When Nrf2 is activated, it drives the expression of $\mathrm{Nrf} 2$ target genes. Therefore, we examined the effects of CZ-7 treatment on the expression of HO-1 and NQO1. As shown in Fig. 10c, d, the protein levels of HO-1 and NQO1 in the hippocampus were up-regulated in the $2 \mathrm{VO}$ group compared with the sham group, and CZ-7 $20 \mathrm{mg} / \mathrm{kg}$ significantly up-regulated these proteins over the model group. These results indicate that CZ-7 treatment could activate the cytoprotective Nrf2 target genes.

\section{DISCUSSION}

Our previous series of studies confirmed the neuroprotective effect of Clau $F$ in vitro. As one of the new derivatives of Clau F, our results first demonstrated that CZ-7 significantly improved cognitive deficit in rats induced by chronic cerebral hypoperfusion. Our current results demonstrate the following: (1) CZ-7 alleviated 2VO-induced memory impairment by shortening the escape latency, increasing the number of crossings and extending the mean time spent in the target quadrant in the MWM test; (2) chronic treatment with $\mathrm{CZ}-7$ in the $2 \mathrm{VO}$ model profoundly reduced brain hippocampus neuronal damage and white matter damage; (3) CZ-7 reduced the destruction indicated by $8-\mathrm{OHdG}$; (4) CZ-7 scavenged the overproduced ROS; and (5) CZ-7 increased Nrf2 entry to the nucleus and enhanced the expression of Nrf2, HO-1, and NQO1. We used three dosages of CZ-7 (10, 20, and 40 $\mathrm{mg} / \mathrm{kg}$ ) for this study, and we observed that the $20 \mathrm{mg} / \mathrm{kg}$ dosage of CZ-7 possessed maximum activity against 2VO-induced VD. Taken together, our findings suggest that the neuroprotective action of CZ-7 is due to its inhibition of oxidative damage through the Nrf2-mediated antioxidant response.

VD is one of the most common types of dementia in older adults, characterized by a progressive worsening of memory and other cognitive functions [1]. In contrast to stroke, which is often caused by a sudden disruption of the blood supply to distinct brain regions, VD is caused by a moderate but persistent reduction in regional cerebral blood flow (CBF), which impairs memory and contributes to the progression of dementia; the damaged vascular system could also cause the deprivation of oxygen and nutrients to the brain and lead to cell death [26]. The ligation of the bilateral common arteries (2VO) can cause global ischemia in the brain. The $2 \mathrm{VO}$ rat is an established model to investigate the effects of chronic cerebral hypoperfusion on cognitive dysfunction and neurodegenerative process [27]. Soon after the 2VO, the CBF in the cortex and hippocampus drops significantly and gradually starts to recover after approximately 1 week, but the deficit is still obvious at 4 weeks [28]. It is generally concluded that the chronically reduced perfusion period following $2 \mathrm{VO}$ appears to mimic best the pathophysiological process of cerebral hypoperfusion [27]. In addition to VD, the 2VO model has been used to study ischemic white matter injury and ischemic eye disease [27].

Decreased cognition is recognized as one of the most severe and typical features in VD, and the MWM is a standard method to assess spatial leaning ability in rodents [29]. 2VO rats need longer escape latencies than controls in the MWM [30]. In this experiment, we found that $2 \mathrm{VO}$ animals exhibited significant learning and memory deficits during the first MWM test on days 22-26 after 2VO surgery. In the second MWM test, on days 48-52, CZ-7-treated animals had significantly decreased total escape latency, but the CMC-Na-treated 2VO group had no obvious improvement in the total escape latency. The neuron is the basic structural and functional unit of the nervous system, and lesions of neurons in specific brain areas, such as the hippocampus, produced by cerebral hypoperfusion can produce severe memory and learning deficits [5]. The Nissl body is one of the characteristic structures of the cytoplasm of neuronal cell bodies, and the density of Nissl staining in the neuronal cytoplasm is used to evaluate neuronal damage [31]. Nissl staining in this study showed that neurons in the hippocampus CA1 and CA3 areas of the model group degenerated after chronic cerebral hypoperfusion, and CZ-7 ameliorated the cerebral hypoperfusion-induced neuron damage. This observation was consistent with the MWM test results. TUNEL staining also showed that $\mathrm{CZ}-7$ had a neuroprotective effect on hippocampal neurons. The white matter is vulnerable to chronic cerebral hypoperfusion, and Klüver-Barrera staining is a commonly used method to evaluate white matter lesions. Brain white matter damage is one of the common causes of cognitive impairment in the elderly and is also one of the main pathological types of vascular cognitive impairment. Our results confirm that CZ-7 significantly improved brain white matter injury.

The above results preliminarily confirm that $\mathrm{CZ}-7$ treatment improved the cognitive function and significantly increased pyramidal neuron number in the hippocampus. We hypothesized that this improved cognitive function was associated with CZ-7's improvement of oxidative stress. This hypothesis is supported by the following: (1) There is an ample evidence that chronic cerebral hypoperfusion is one of the key factors in the development of cognitive impairment, in which vascular oxidative stress plays a major role [32]. (2) We have confirmed that $\mathrm{CZ}-7$ has a protective effect in $\mathrm{H}_{2} \mathrm{O}_{2}$-induced $\mathrm{PC} 12$ cells and primary neuron cells damage in vitro [15]. (3) CZ-7 exhibits strong free radicalscavenging activity [15]. Redox homeostasis in the human body is a complex process, and ROS are involved in the maintenance of redox homeostasis and various cellular signaling pathways. Increased oxidative stress is a widely accepted participant in the development and progression of both $A D$ and VD [33, 34]. Oxidative stress plays a key role in neuronal death during cerebral hypoperfusion and may deteriorate cognitive function in cerebrovascular disease [35]. The oxidative damage to DNA, lipids, and proteins can produce $8-\mathrm{OHdG}$, 4-hydroxynonenal (4-HNE), and nitrotyrosine (NTY), respectively [36]. Oxidative damage of DNA is one of the most important mechanisms for the pathogenesis of cancer, cardiovascular, and other chronic diseases. [37, 38] 8$\mathrm{OHdG}$, as a biomarker of oxidative damage in DNA, is a promising indicator for these diseases, and the extent of oxidative damage and repair in vivo can be assessed by $8-O H d G$ detection [39]. Reactive oxygen radicals, such as hydroxyl radicals and superoxide anions, attack the guanine eighth-base carbon atoms in DNA molecules, which can form the oxidative adduct $8-\mathrm{OHdG}$, thus changing the spatial structure of DNA chains [40]. Increased urinary $8-\mathrm{OHdG}$ has been associated with lower cognitive performance [41] and is a potential marker of oxidative stress in VD patients [42]. Under physiological conditions, the antioxidant system of the body can promptly remove excess free radicals, maintaining the redox balance; but in pathological conditions, ROS are quickly released and excessively accumulate in the body, leading to oxidative stress that exceeds the antioxidant capacity of the body, which then causes oxidative damage to DNA and further leads to the release of $8-\mathrm{OHdG}$ [43]. Its high rate of oxygen consumption, its richness in fatty acids, and 


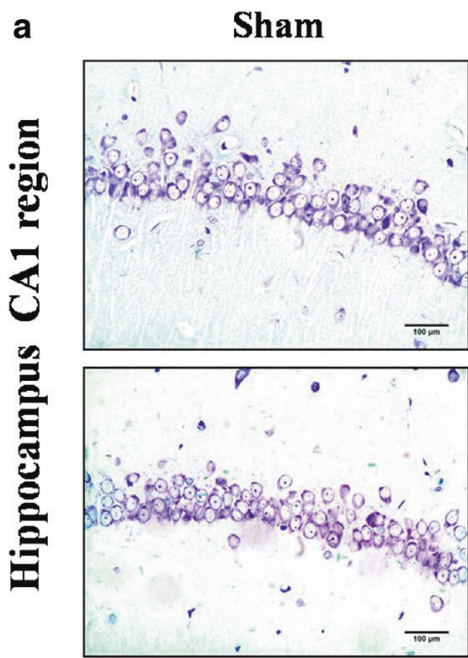

CZ-7-20 mg/kg

b
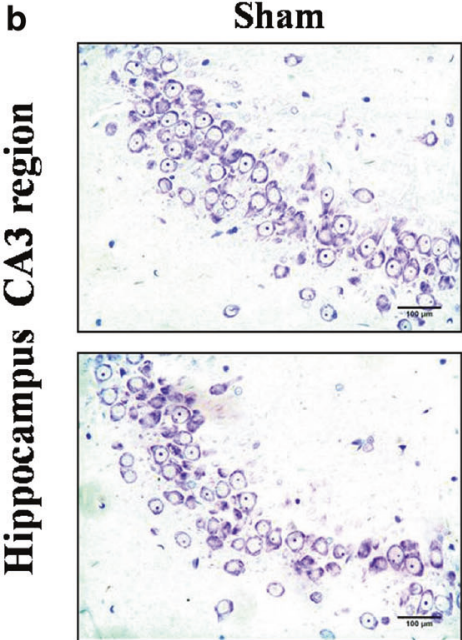

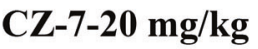

Model
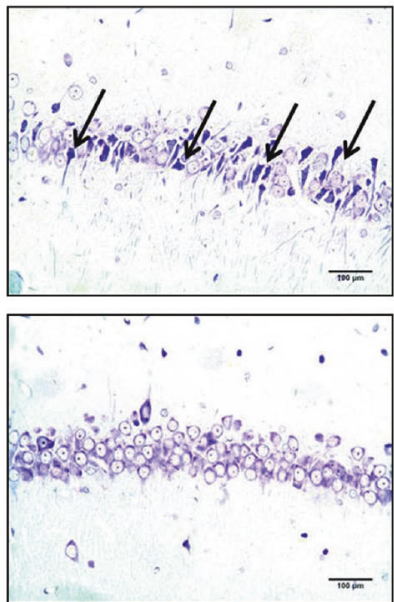

$\mathrm{CZ-7-40} \mathrm{mg/kg}$

Model
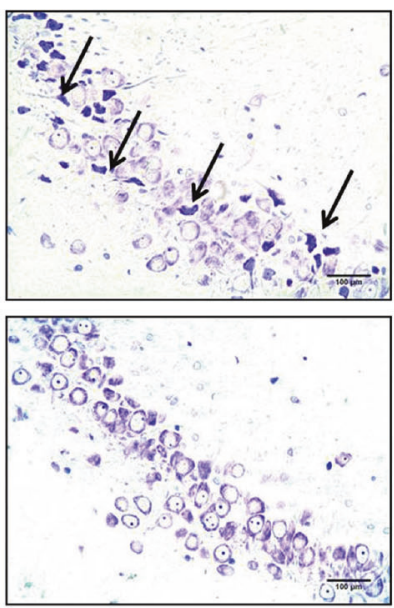

$\mathrm{CZ}-7-40 \mathrm{mg} / \mathrm{kg}$
CZ-7-10 mg/kg
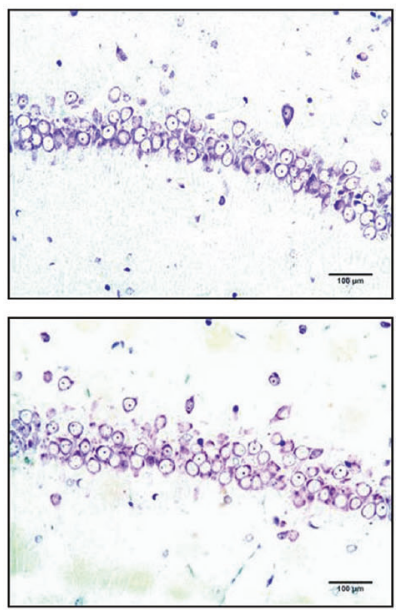

Nimodipine

CZ-7-10 mg/kg
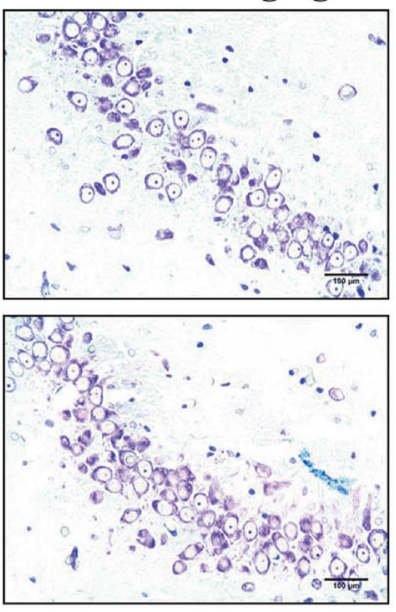

Nimodipine

C

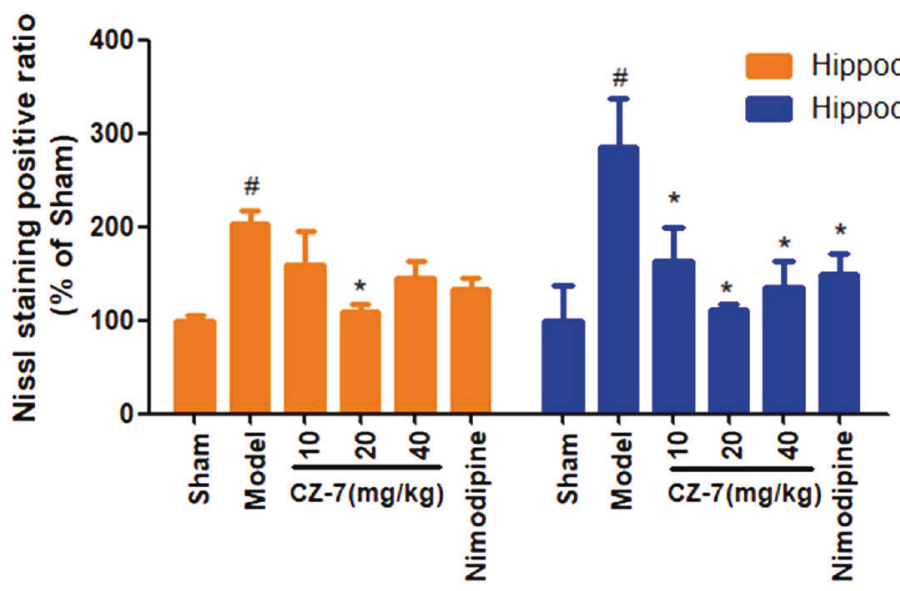

Fig. 4 a, b The effects of CZ-7 on the morphologic changes in the hippocampal CA 1 and CA3 regions induced by chronic cerebral hypoperfusion in rats (Nissl staining). Representative micrographs: $\times 200$ magnification, scale bar $=100 \mu \mathrm{m}$. The black arrows point to the damaged Nissl bodies of neurons. c Quantification of CZ-7's effects on neuron damage. Values are expressed as the mean \pm SEM $(n=3) .{ }^{\#} P<0.05$, vs. sham group, ${ }^{*} P<0.05$, vs. model group. For CA1, one-way ANOVA, $F(5,16)=3.897, P=0.0281$. For CA3, one-way ANOVA, $F(5,17)=3.998, P=0.0229$ 

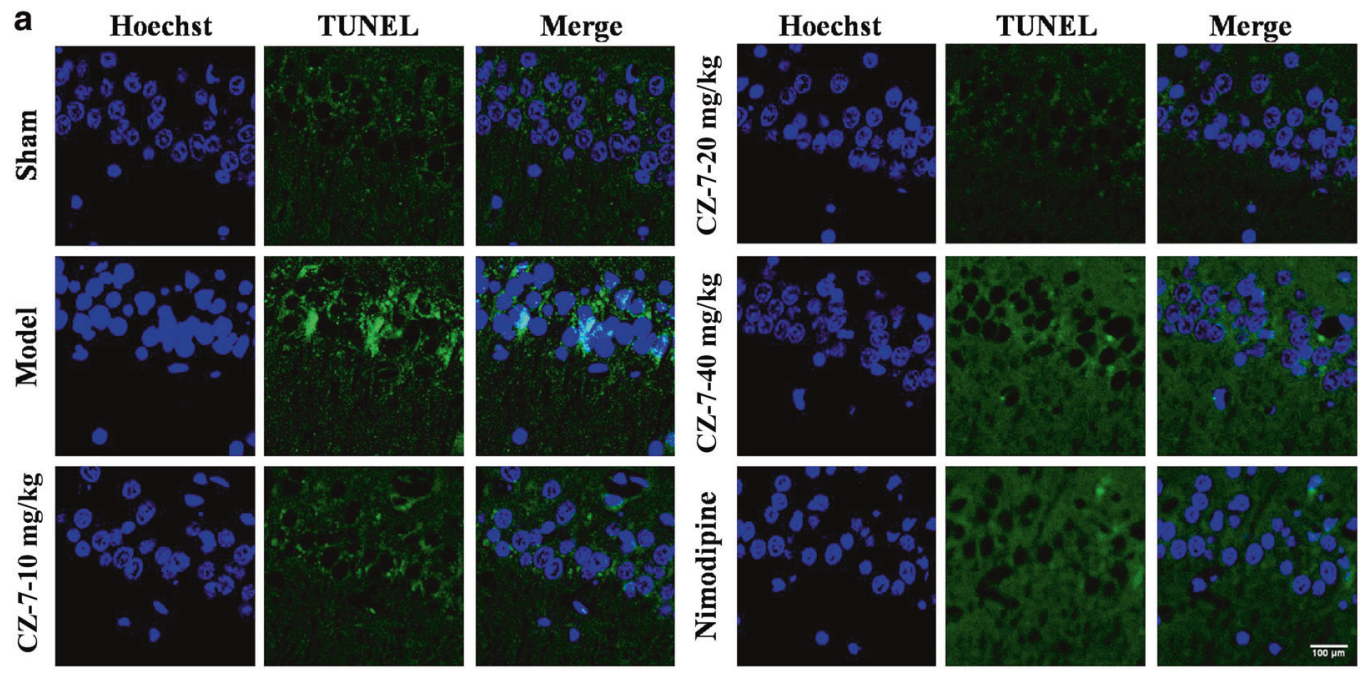

TUNEL
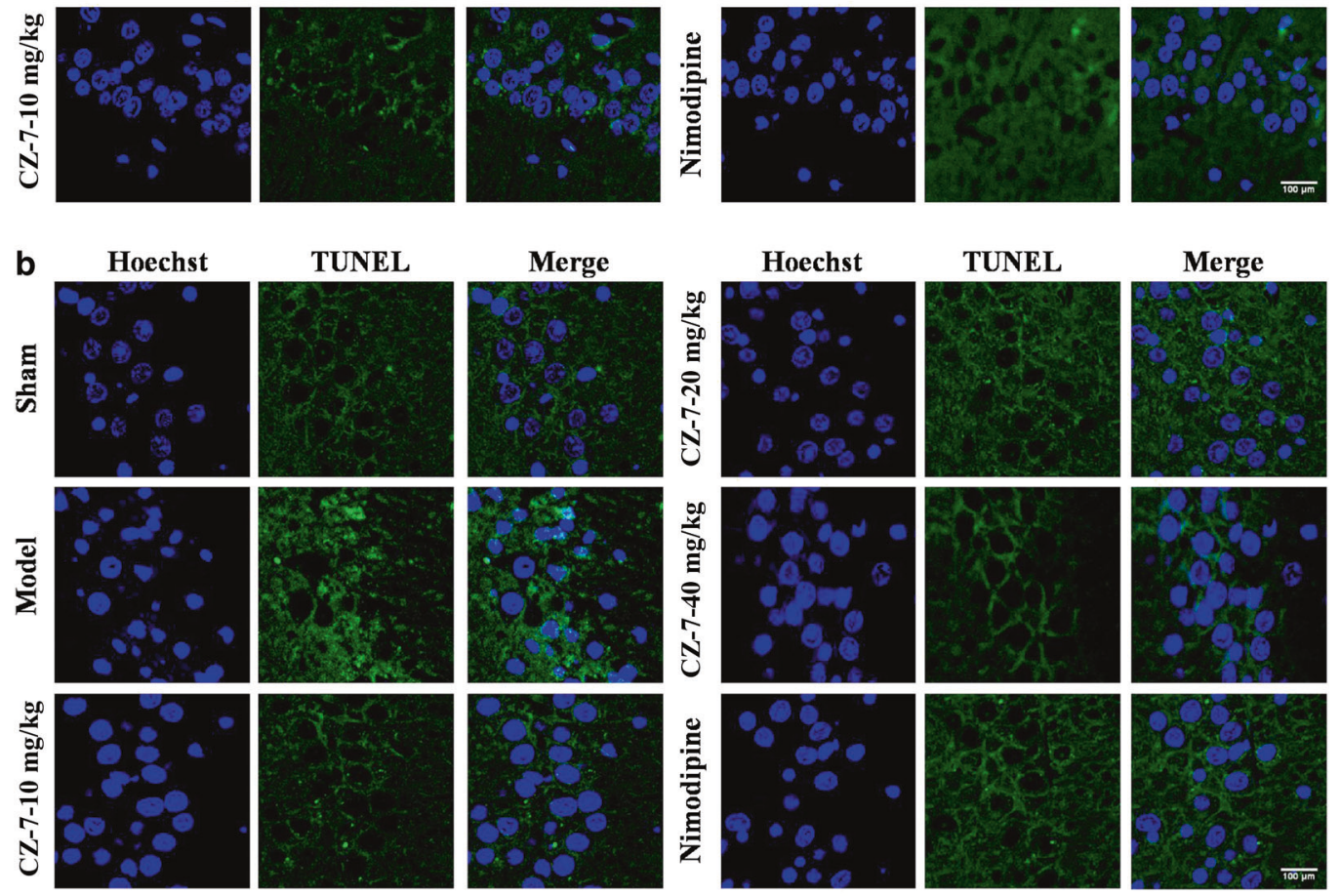

C

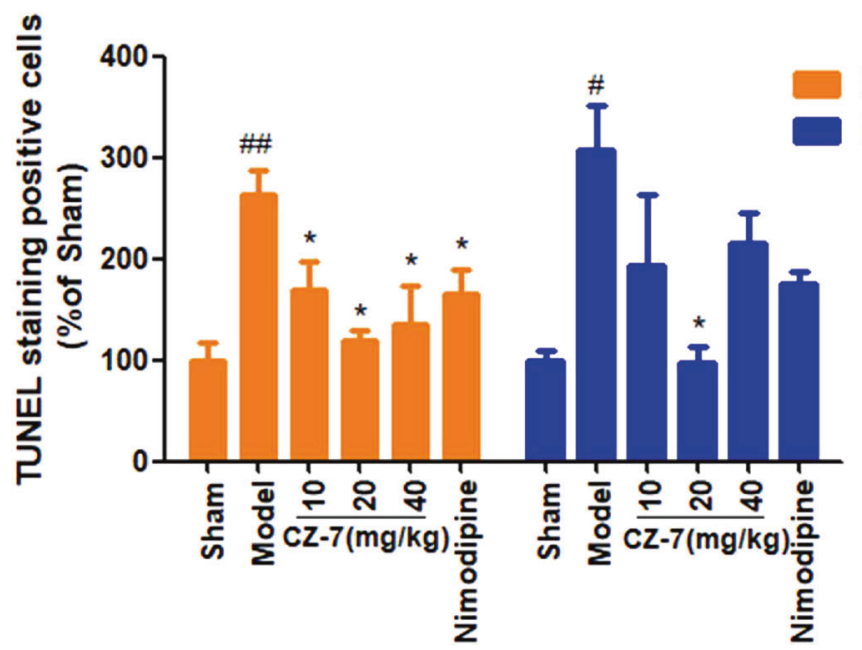

Hippocampal CA1 region

Hippocampal CA3 region

Fig. 5 a, b The effects of CZ-7 on apoptosis in the hippocampal CA1 and CA3 regions induced by chronic cerebral hypoperfusion in rats (TUNEL staining). Representative micrographs: $\times 200$ magnification, scale bar $=100 \mu \mathrm{m}$. c Quantification of CZ-7's effect on apoptosis-positive neurons. Values are expressed as the mean \pm SEM $(n=3)$. ${ }^{\#} P<0.05,{ }^{\#} P<0.01$ vs. sham group. ${ }^{*} P<0.05$ vs. model group. For $C A 1$, one-way ANOVA, $F(5,17)=5.451, P=0.076$. For CA3, one-way ANOVA, $F(5,17)=4.407, P=0.0164$ 
a
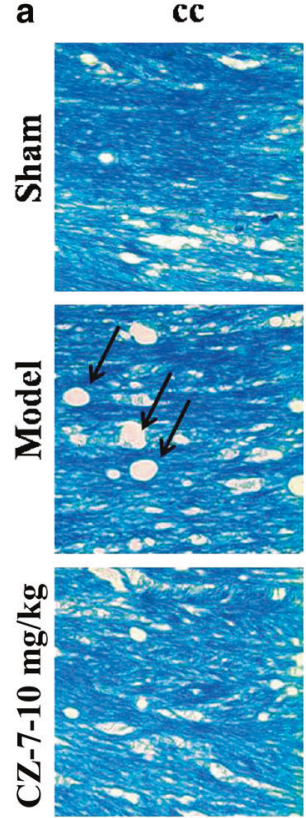

ic
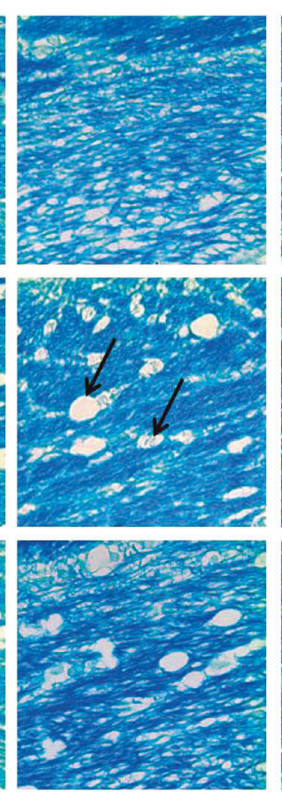
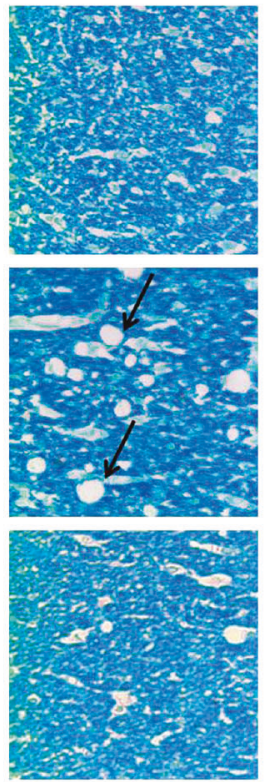

cc
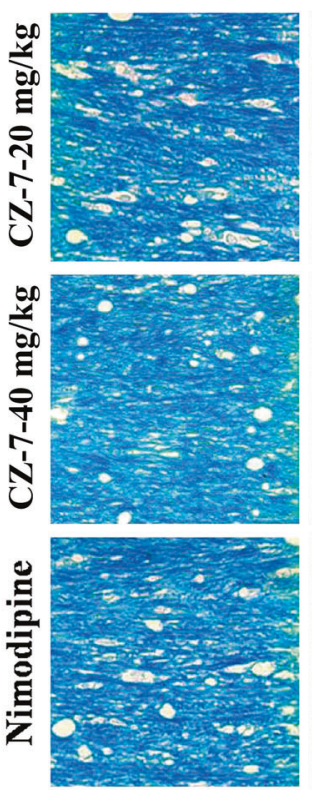

ec
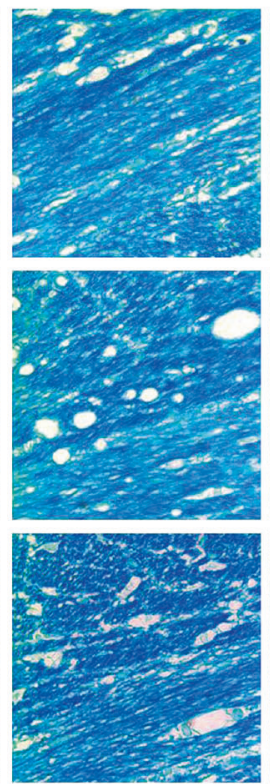

ic
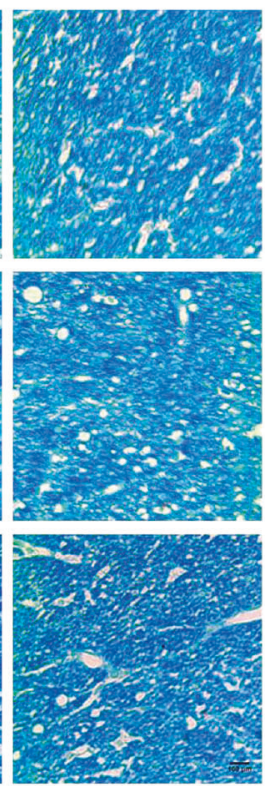

b

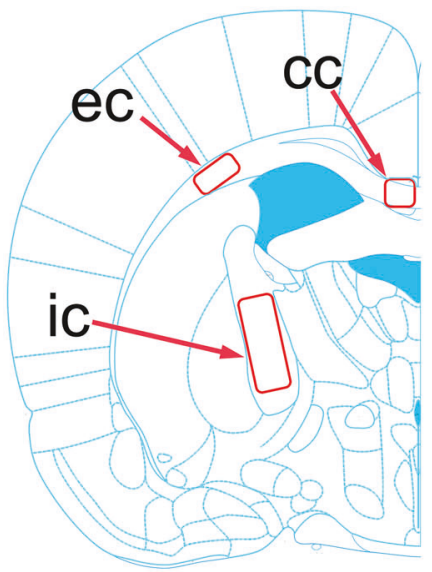

Interaural $7.68 \mathrm{~mm}$

C

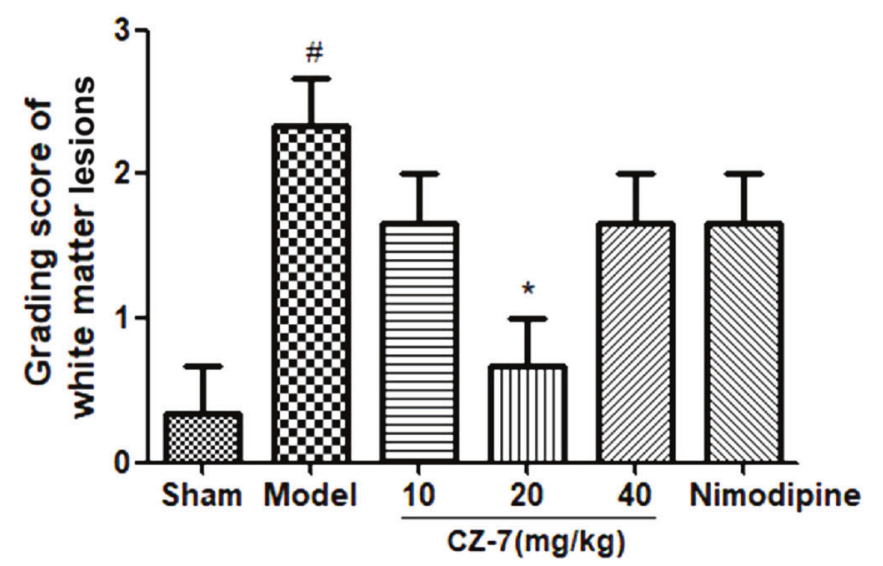

Fig. 6 Effects of CZ-7 on chronic cerebral hypoperfusion-induced white matter lesions. a Representative photographs of tissue sections stained with Klüver-Barrera in the myelinated fibers of the corpus callosum (cc), external callosum (ec), and internal capsule (ic). Arrows indicate the formation of marked vacuoles and the disappearance of myelinated fibers. $\mathbf{b}$ A schematic elucidation of the rat cc, ec, and ic. Red boxes represent the observed areas of all brain slices in a. c Grading score of white matter lesion. Values are expressed as mean \pm SEM. $n=3$. ${ }^{\#} P<0.05$ vs. Sham group, ${ }^{*} P<0.05$ vs. Model group. For grading score of white matter lesion, one-way ANOVA, $F(5,17)=4.967, P=0.0107$

its weak antioxidant activity [44] render the brain especially sensitive to oxidative stress, so an imbalanced antioxidant system will contribute to neuronal cell loss and exacerbate VD [45]. As reflected in Fig. 5a and $b$, the results of $8-O H d G$ immunofluorescence showed that the number of $8-\mathrm{OHdG}$ positive cells in the model group increased significantly. Longterm administration of CZ-7 effectively improved the oxidative stress status and reduced the number of positive cells in the hippocampus. The results of ROS immunofluorescence are consistent with 8-OHdG.

Nrf2 is a member of the cap-n-collar transcription factor family, and it is a key player in the cellular antioxidant defense system. Nrf2 is considered a potential target to prevent oxidative damage because its activation protects different organs from ischemia/reperfusion injury. Nrf2-deficient female mice are very sensitive to oxidative stress, and they develop a severe lupus-like glomerulonephritis, a type of autoimmune disease that is closely related to increasing oxidative stress [46]. Nrf2 has emerged as a new therapeutic target. Inhibitors of Nrf2 are expected to help reduce drug resistance and improve therapy, and activators of Nrf2 are helpful in protection against drug toxicity and stress-induced diseases [47, 48]. However, one of the problems that need to be taken into consideration is that the role of Nrf2 is complicated, even in the same disease. For example, Nrf2 promotes cancer cell survival and confers resistance to chemotherapeutics and radiotherapy [49]. By disassociating from Keap1 and translocating from the cytoplasm to the nucleus, Nrf2 has antioxidant effects. Nrf2 is involved in upregulating a battery of antioxidant and cellprotective genes, including NQO1 and $\mathrm{HO}-1$ [50]. As reflected in our results, CZ-7 can effectively promote the Nrf2 translocation from the cytosol to the nucleus. NQO1 and $\mathrm{HO}-1$ were 

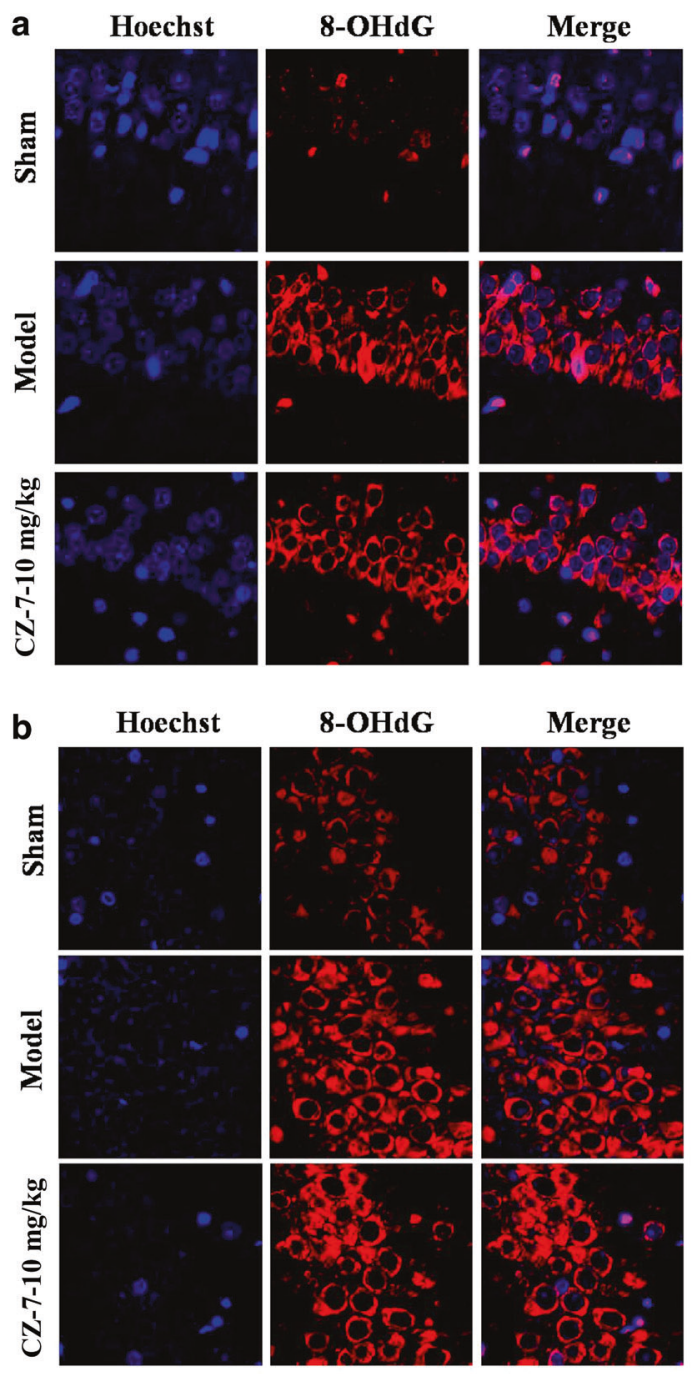

\section{8-OHdG}
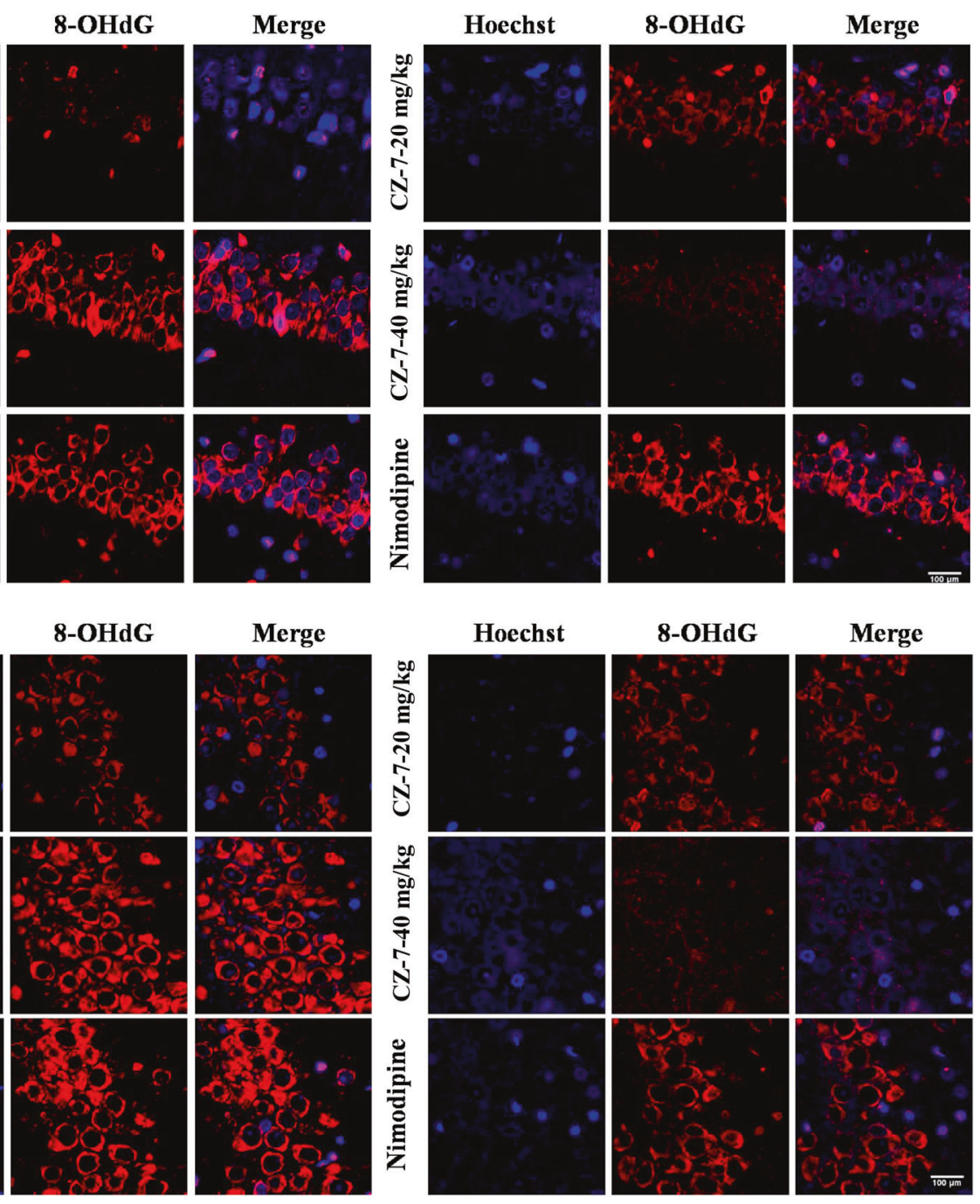

C

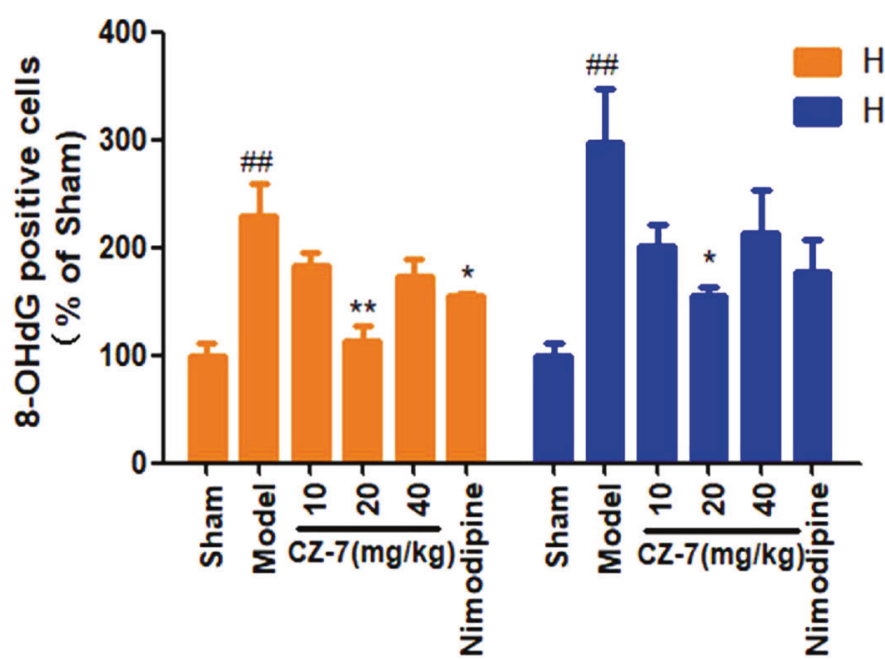

Fig. 7 a, b The effects of CZ-7 on 8-OHdG changes in hippocampal CA1 and CA3 region induced by chronic cerebral hypoperfusion in rats. Representative micrographs: $\times 200$ magnification, scale bar $=100 \mu \mathrm{m}$. c Quantification analysis of CZ-7 on 8-OHdG-positive cells. Values are expressed as mean \pm SEM. $n=3$. ${ }^{\# \#} P<0.01$ vs. Sham group, ${ }^{*} P<0.05,{ }^{* *} P<0.01$ vs. Model group. For CA1, one-way ANOVA, $F(5,17)=8.424$, $P=0.0013$. For CA3, one-way ANOVA, $F(5,17)=4.667, P=0.0134$ 

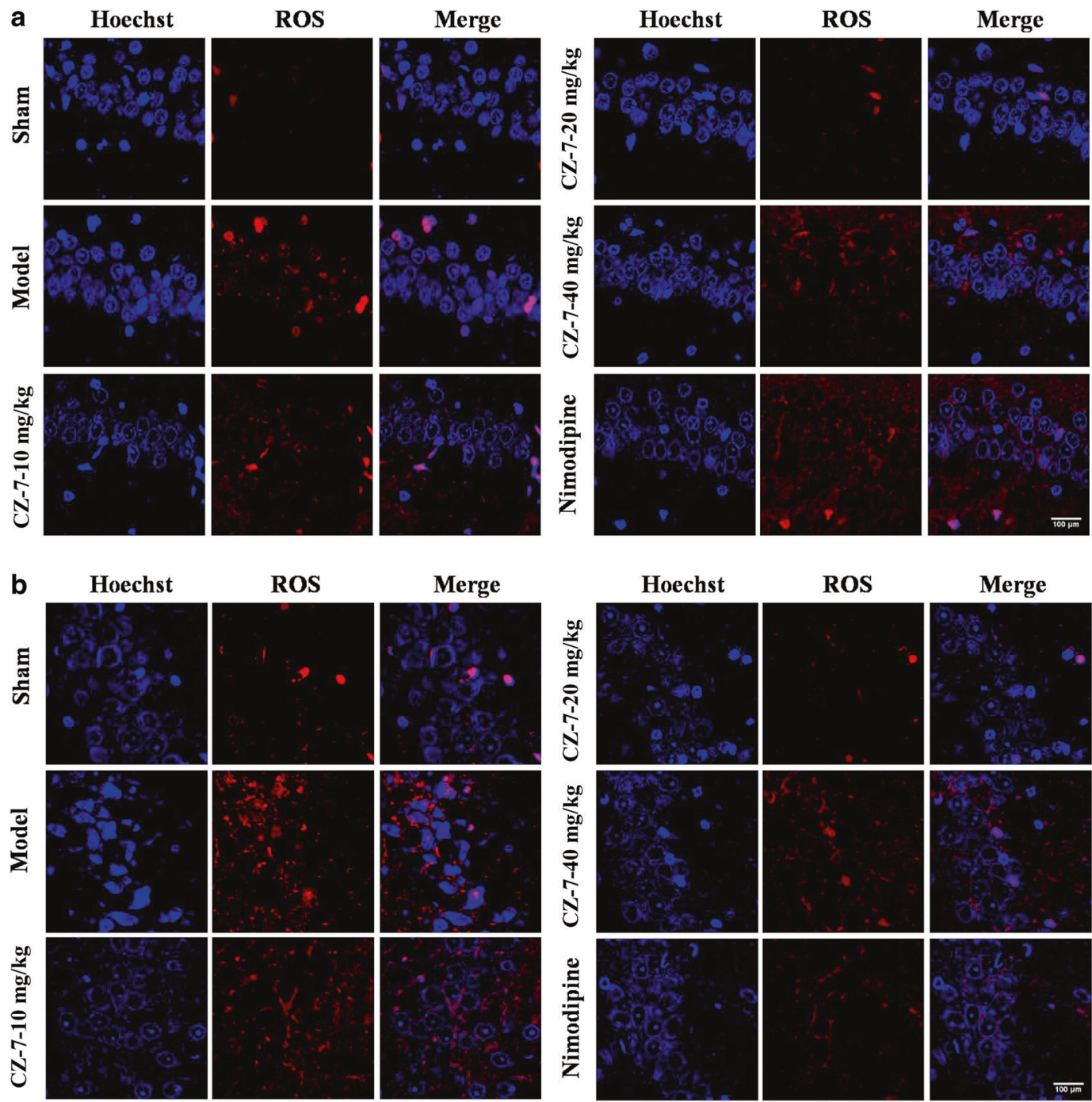

c

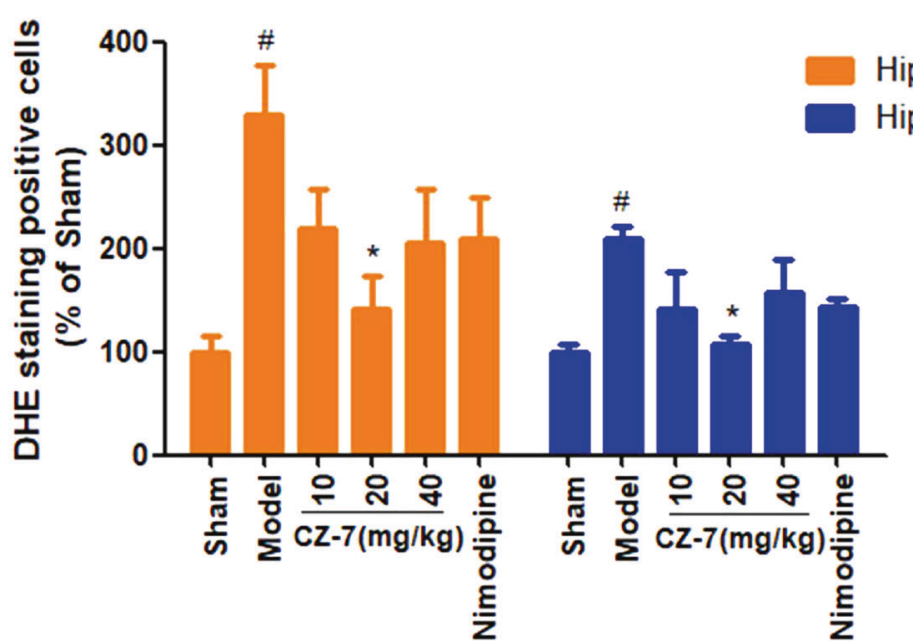

Fig. 8 a, b The effects of CZ-7 on ROS changes in hippocampal CA1 and CA3 region induced by chronic cerebral hypoperfusion in rats. Representative micrographs: $\times 200$ magnification, scale bar $=100 \mu \mathrm{m}$. c Quantification of CZ-7 effect on ROS-positive cells. Values are expressed as mean \pm SEM. $n=3$. ${ }^{\#} P<0.05$ vs. Sham group. ${ }^{*} P<0.05$ vs. Model group. For CA1, one-way ANOVA, $F(5,17)=3.841, P=0.0261$. For CA3, one-way ANOVA, $F(5,17)=3.411, P=0.0379$ 

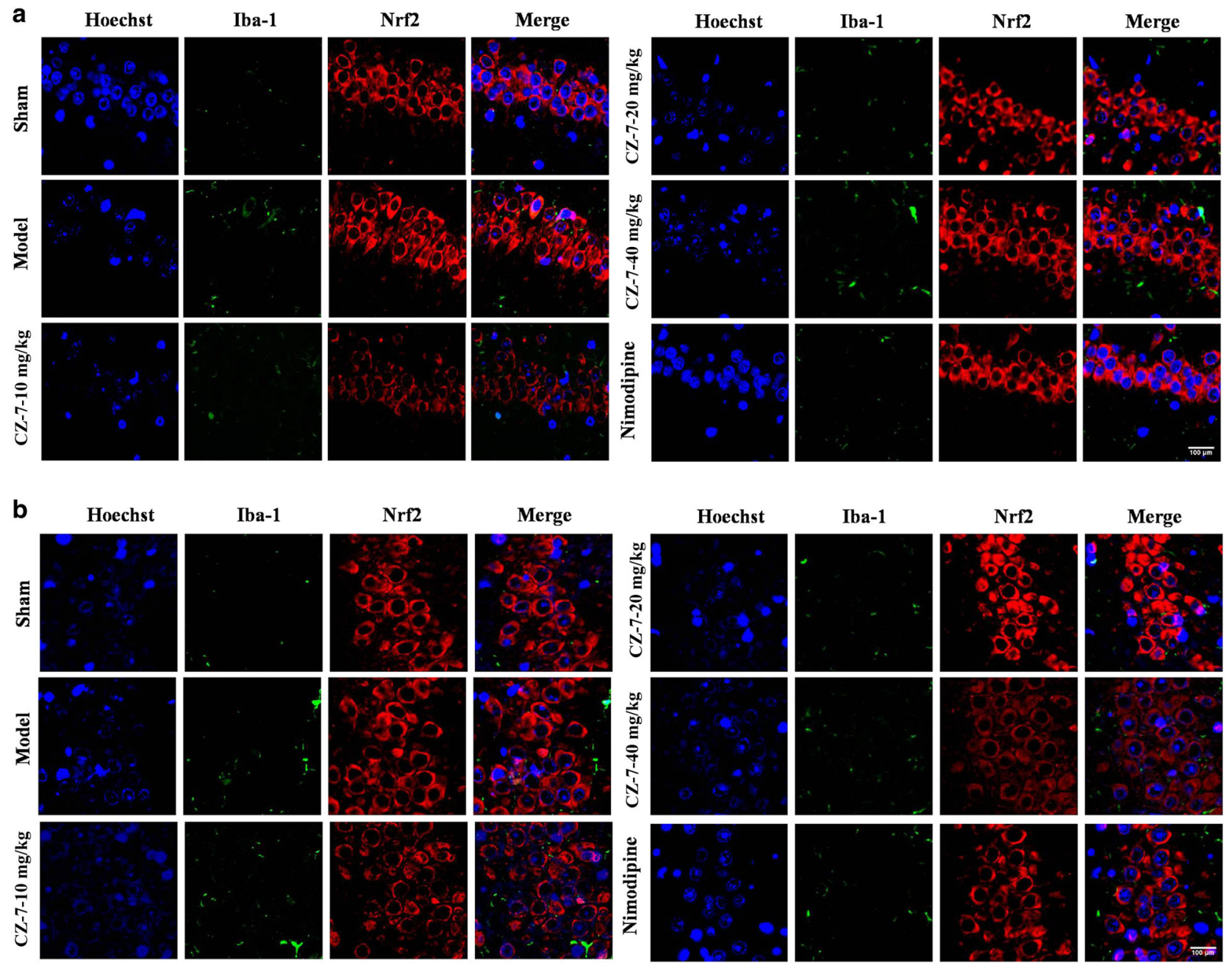

C Hoechst
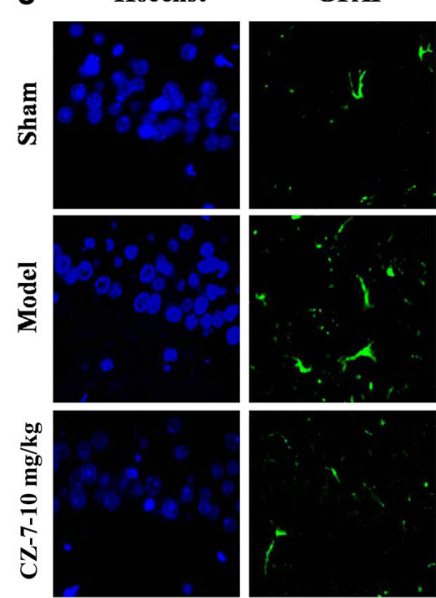

Nrf2

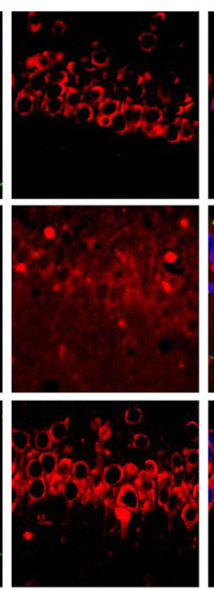

Merge
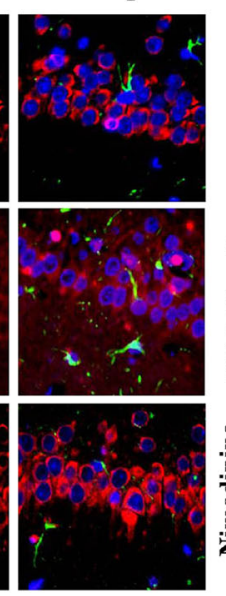

Hoechst
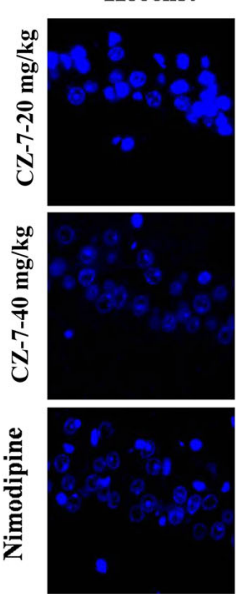

GFAP

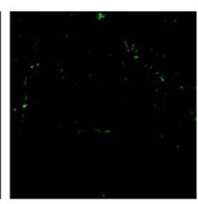

Nrf2

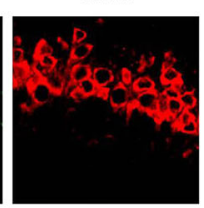

Merge

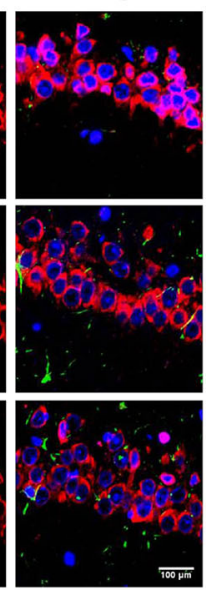

up-regulated in the CZ-7 treatment groups, particularly in the CZ-7 $20 \mathrm{mg} / \mathrm{kg}$ group.

\section{CONCLUSION}

CZ-7 significantly improved the cognitive deficits induced by chronic cerebral hypoperfusion in rats. Our investigation on the mechanism of the neuroprotective effect of CZ-7 showed that
CZ-7 $(20 \mathrm{mg} / \mathrm{kg})$ significantly increased the surviving pyramid cells of the CA1-CA3 area of the hippocampus of $2 \mathrm{VO}$ rats, alleviated brain white matter injury, decreased the cellular levels of 8-OHdG and ROS, and enhanced the antioxidant capacity by upregulating HO-1 and NQO1 through Nrf2 activation. These effects might be related to its antioxidation activities, but the exact target of CZ-7 still needs to be determined. In this regard, $\mathrm{Nrf2}^{-} /^{-}$mice may be helpful to verify its exact target. 

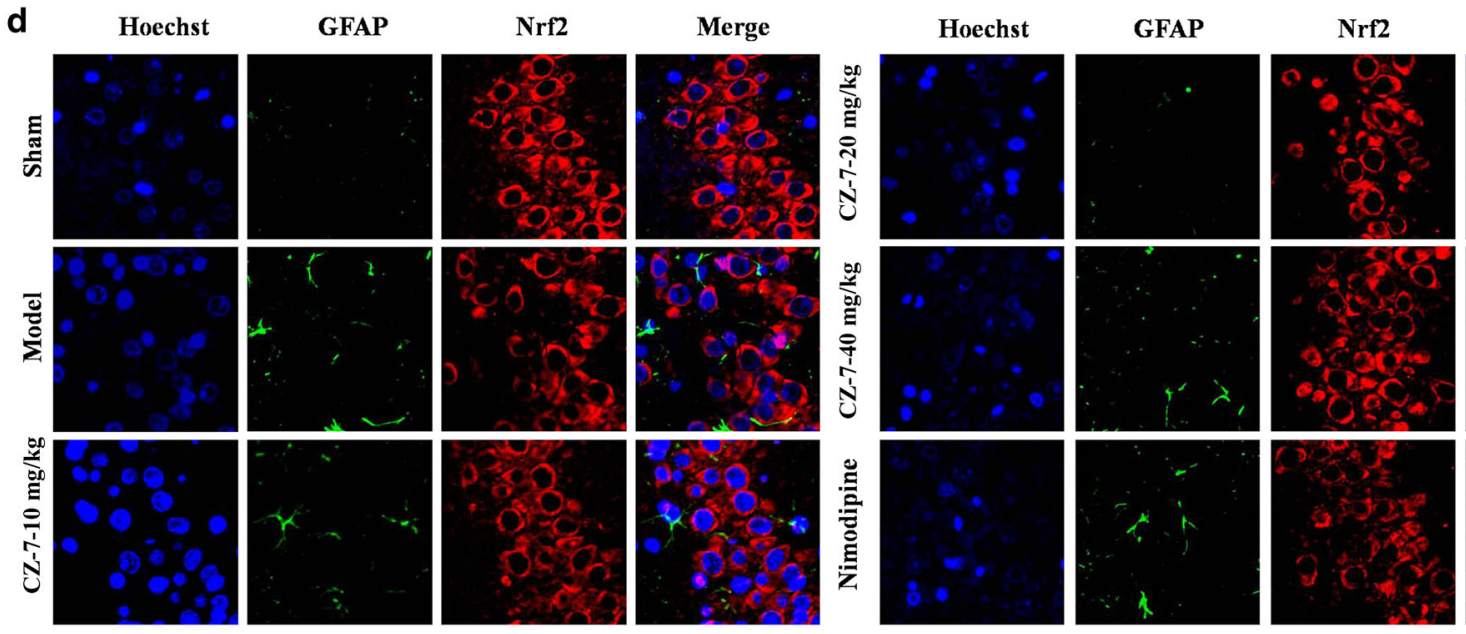

Merge
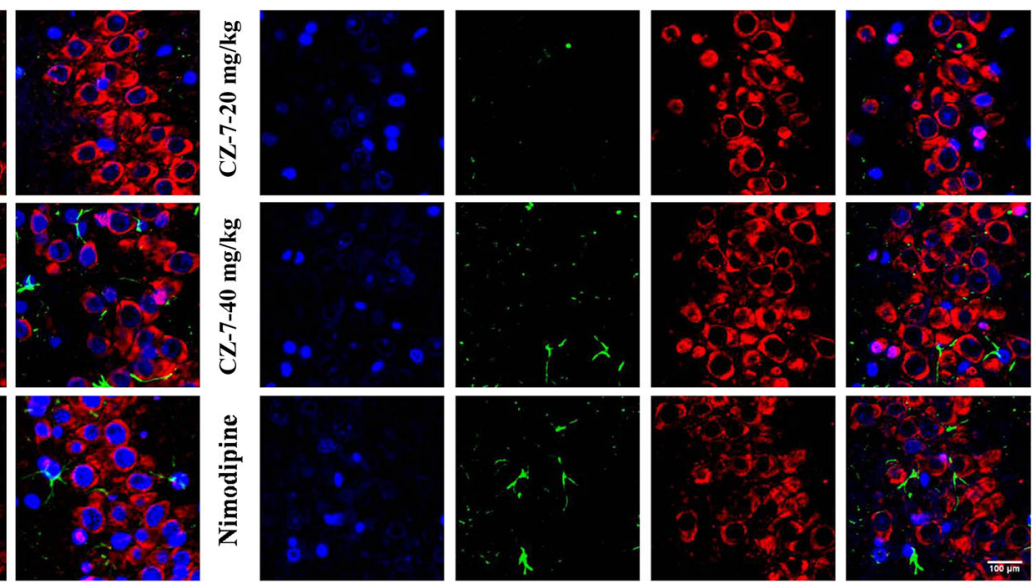

e Hoechst

Nrf2

Merge

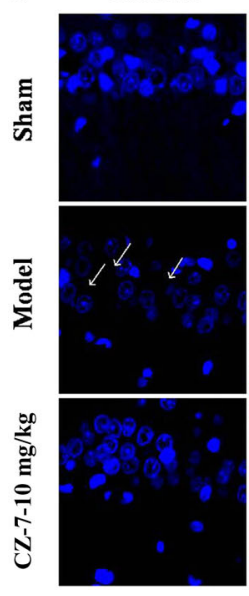

MAP2
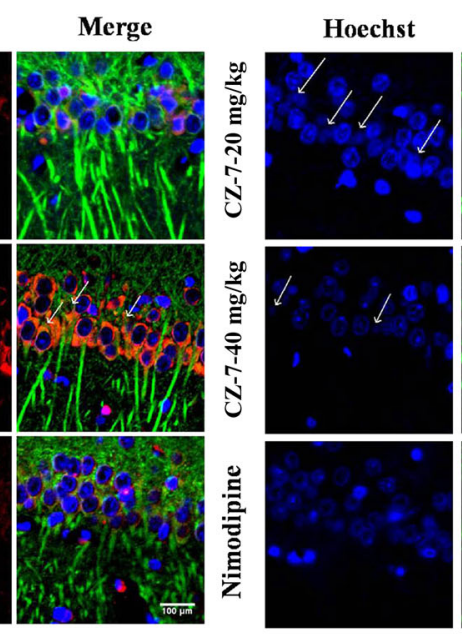

MAP2

Nrf2
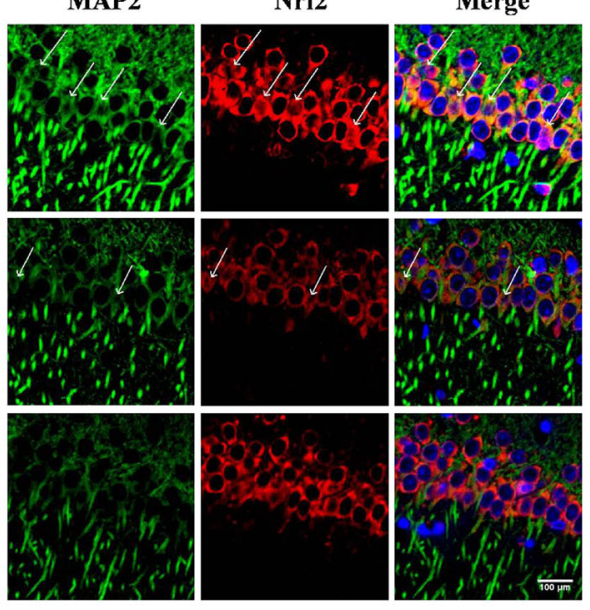

\section{f Hoechst}
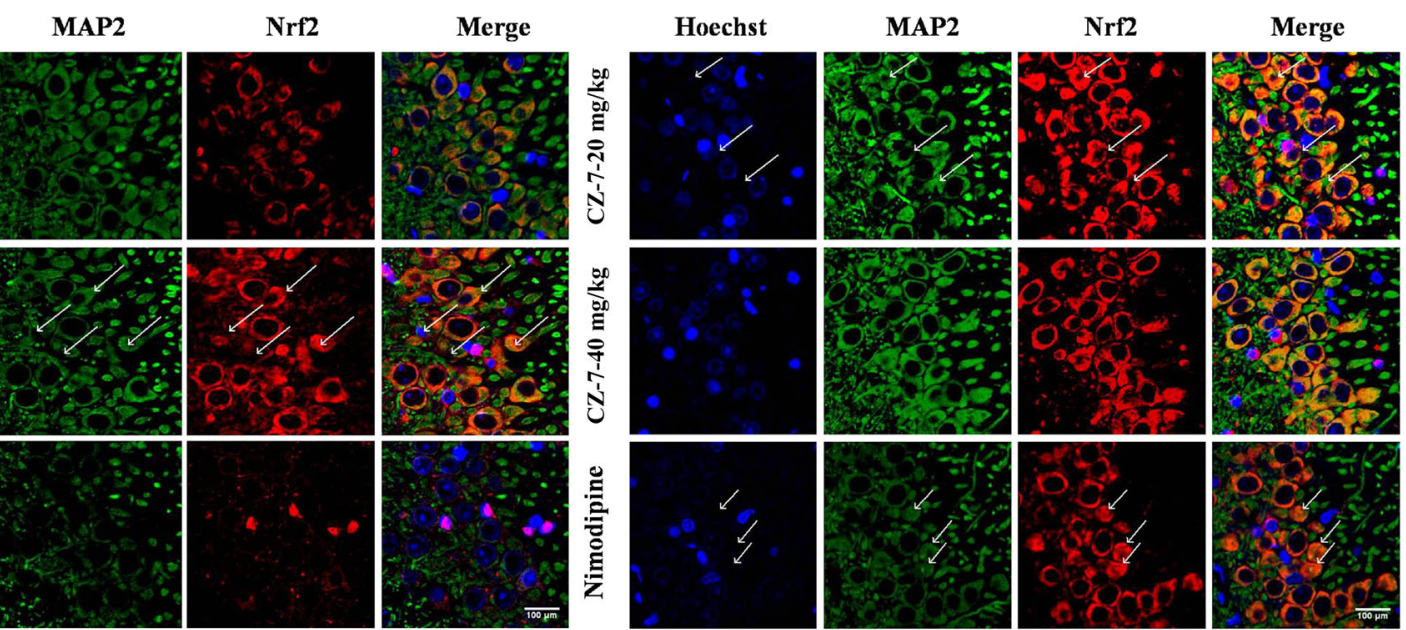

Fig. 9 Colocalization of Nrf2 and lba-1, GFAP or MAP2 in the rat hippocampus by immunofluorescence. $\mathbf{a}, \mathbf{b}$ The effects of CZ-7 on the levels of Nrf2 in hippocampus CA1 and CA3 region of rats based on double immunofluorescence assay with lba-1. c, d The effects of CZ-7 on the levels of Nrf2 in hippocampus CA1 and CA3 region of rats based on double immunofluorescence assay with GFAP. e, $\mathbf{f}$ The effects of CZ-7 on the levels of Nrf2 in hippocampus CA1 and CA3 region of rats based on double immunofluorescence assay with MAP2. Representative micrographs: $\times 200$ magnification, scale bar $=100 \mu \mathrm{m}$. The white arrows indicate the Nrf2 translocation from the cytoplasm to the nucleus 

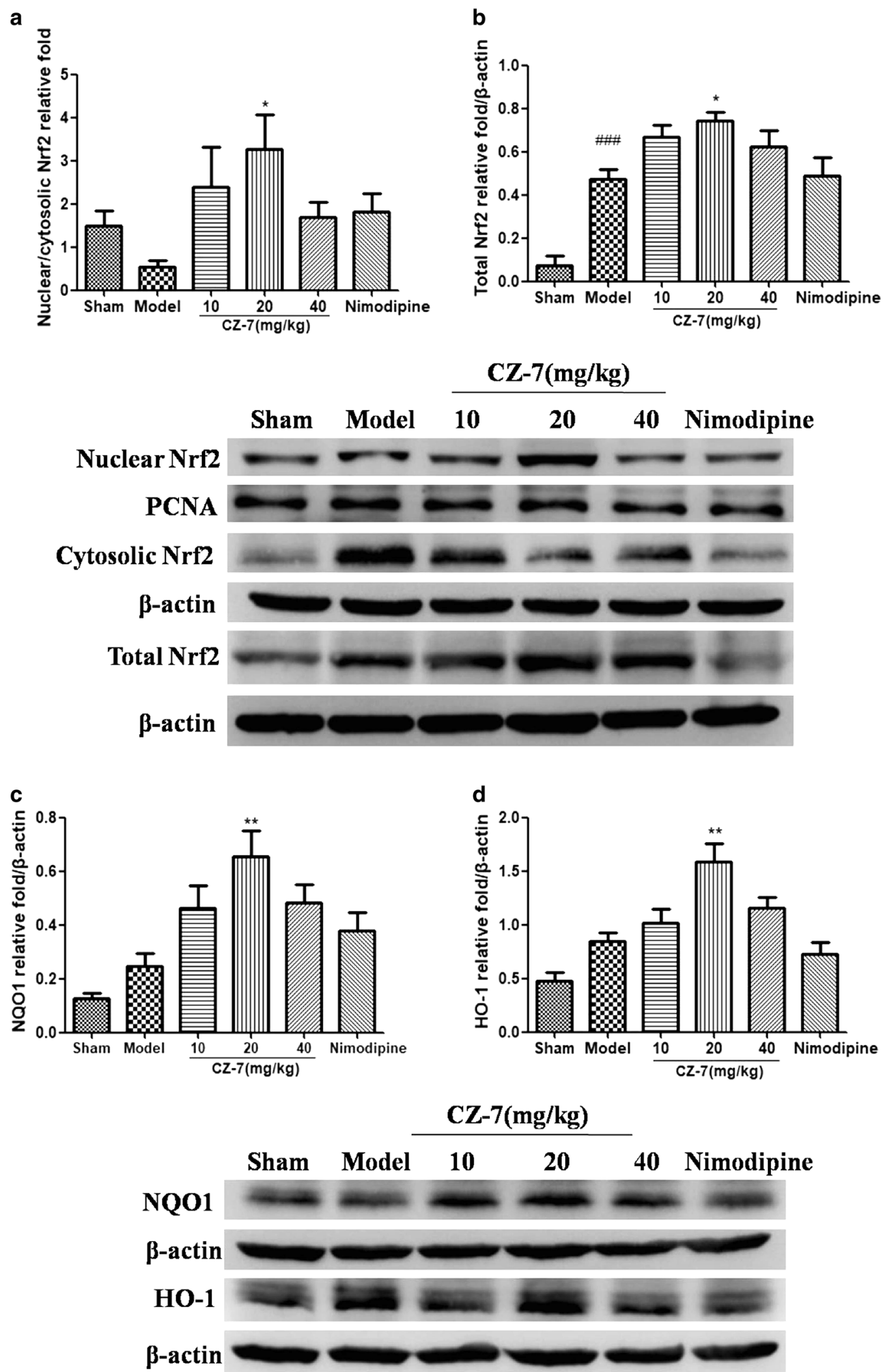

Fig. 10 CZ-7 triggered the activation of Nrf2 and its target genes. $\mathbf{a}$, b Representative immunoblots and densitometry analysis of Nrf2 in the nuclear/cytosolic relative fold and in the total protein of hippocampus. c, d Representative immunoblots and densitometry analysis of NQO1 and HO-1 in the total protein of hippocampus. Values are expressed as mean \pm SEM. $n=4$ for each group. ${ }^{\# \# \#} P<0.001$ vs. Sham group. ${ }^{*} P<$ $0.05 * * P<0.01$ vs. Model group. For Nrf2 in the nuclear/cytosolic relative fold, one-way ANOVA, $F(5,18)=2.540, P=0.0659$. For Nrf2 in the total protein, one-way ANOVA, $F(5,22)=12.46, P<0.0001$. For NQO1, one-way ANOVA, $F(5,22)=7.909, P=0.0005$. For HO-1, one-way ANOVA, $F(5,23)=10.73, P<0.0001$ 


\section{ACKNOWLEDGEMENTS}

This work was supported by the National Natural Science Foundation of China (Nos. 81730096, 81730093, U1402221, 81603316, 81560685), the CAMS Innovation Fund for Medical Sciences (No. CIFMS) (No. 2016-12M-1-004), the State Key Laboratory Fund Open Project (No. GTZK201610), the China Postdoctoral Science Foundation (No. 2013M540066), the PUMC Graduate Education and Teaching Reform Project (No. 10023201600801), the Scientific Research Foundation of the Higher Education Institutions of Hunan Province (No. 15K091), the Project of NDRC and State Administration of Traditional Chinese Medicine (No. 60011000), and the Hunan Provincial Key Laboratory for Standardization of Important Chinese Herbal Pieces (No. BG201701, 4981-0901020).

\section{AUTHOR CONTRIBUTION}

$\mathrm{N}$-hC, S-fC, XH, Z-hH, H-sS, and Z-pF designed research; D-dL, XY, CC, QR, PL, M-yL, S$\mathrm{sW}, \mathrm{T}-\mathrm{bZ}$ and Q-dA performed research; $\mathrm{Y}-\mathrm{dZ}$ and D-mZh contributed new analytical tools and reagents; D-dL and $\mathrm{XY}$ analyzed data; D-dL and XY wrote the paper.

\section{ADDITIONAL INFORMATION}

Competing interests: The authors declare no competing interests.

\section{REFERENCES}

1. Kua EH, Ho E, Tan HH, Tsoi C, Thng C, Mahendran R. The natural history of dementia. Psychogeriatrics. 2014;14:196-201.

2. O'Brien JT, Thomas A. Vascular dementia. Lancet. 2015;386:1698-706.

3. Sahathevan R, Brodtmann A, Donnan GA. Dementia, stroke, and vascular risk factors; a review. Int J Stroke. 2012;7:61-73.

4. Korczyn AD, Vakhapova V, Grinberg LT. Vascular dementia. J Neurol Sci. 2012;322:2-10.

5. Venkat $\mathrm{P}$, Chopp M, Chen J. Models and mechanisms of vascular dementia. Exp Neurol. 2015;272:97-108.

6. Wang J, Zhang HY, Tang XC. Cholinergic deficiency involved in vascular dementia: possible mechanism and strategy of treatment. Acta Pharmacol Sin. 2009;30:879-88.

7. Versijpt J. Effectiveness and cost-effectiveness of the pharmacological treatment of Alzheimer's disease and vascular dementia. J Alzheimer Dis. 2014;42(Suppl 3): S19-25.

8. Dichgans M, Leys D. Vascular cognitive impairment. Circ Res. 2017;120:573-91.

9. Chu SF, Zhang JT. Recent advances in the study of (-)clausenamide: chemistry, biological activities and mechanism of action. Acta Pharm Sin B. 2014;4:417-23.

10. Chu S, Liu S, Duan W, Cheng $Y$, Jiang $X$, Zhu $C$, et al. The anti-dementia drug candidate, (-)-clausenamide, improves memory impairment through its multitarget effect. Pharmacol Ther. 2016;162:179-87.

11. Liu H, Li CJ, Yang JZ, Ning N, Si YK, Li L, et al. Carbazole alkaloids from the stems of Clausena lansium. J Nat Prod. 2012;75:677-82.

12. Li JW, Ning N, Ma YZ, Zhang R, Tan F, Chen NH. Claulansine F suppresses apoptosis induced by sodium nitroprusside in PC12 cells. Free Radic Res. 2013;47:488-97.

13. Ma YZ, Ning N, He WB, Li JW, Hu JF, Chu SF, et al. Claulansine F promotes neuritogenesis in PC12 cells via the ERK signaling pathway. Acta Pharmacol Sin. 2013;34:1499-507.

14. Huang JY, Ma YZ, Yuan YH, Zuo W, Chu SF, Liu H, et al. Claulansine F promoted the neuronal differentiation of neural stem and progenitor cells through Akt/ GSK-3beta/beta-catenin pathway. Eur J Pharmacol. 2016;786:72-84.

15. Zang Y, Song X, Li C, Ma J, Chu S, Liu D, et al. Pyrano[3,2-a]carbazole alkaloids as effective agents against ischemic stroke in vitro and in vivo. Eur J Med Chem. 2017;143:438-48.

16. Ji HJ, Hu JF, Wang YH, Chen XY, Zhou R, Chen NH. Osthole improves chronic cerebral hypoperfusion induced cognitive deficits and neuronal damage in hippocampus. Eur J Pharmacol. 2010;636:96-101.

17. Zhao XL, Fang XB, Li DP. Establishing vascular dementia model in rats. J Chin Med Univ. 2002;31:166-7.

18. He XL, Wang $\mathrm{YH}$, Bi MG, Du GH. Chrysin improves cognitive deficits and brain damage induced by chronic cerebral hypoperfusion in rats. Eur J Pharmacol. 2012;680:41-8.

19. Zhang T, Gu J, Wu L, Li N, Sun Y, Yu P, et al. Neuroprotective and axonal outgrowth-promoting effects of tetramethylpyrazine nitrone in chronic cerebral hypoperfusion rats and primary hippocampal neurons exposed to hypoxia. Neuropharmacology. 2017;118:137-47.

20. Sugawara T, Lewen A, Noshita N, Gasche Y, Chan PH. Effects of global ischemia duration on neuronal, astroglial, oligodendroglial, and microglial reactions in the vulnerable hippocampal CA1 subregion in rats. J Neurotrauma. 2002;19:85-98.

21. Choi BR, Kim DH, Back DB, Kang CH, Moon WJ, Han JS, et al. Characterization of white matter injury in a rat model of chronic cerebral hypoperfusion. Stroke. 2016;47:542-7.

22. Di Minno A, Turnu L, Porro B, Squellerio I, Cavalca V, Tremoli E, et al. 8-Hydroxy-2deoxyguanosine levels and cardiovascular disease: a systematic review and metaanalysis of the literature. Antioxid Redox Signal. 2016;24:548-55.

23. Wu LL, Chiou CC, Chang PY, Wu JT. Urinary 8-OHdG: a marker of oxidative stress to DNA and a risk factor for cancer, atherosclerosis and diabetics. Clin Chim Acta. 2004;339:1-9.

24. Bae YS, Oh H, Rhee SG, Yoo YD. Regulation of reactive oxygen species generation in cell signaling. Mol Cells. 2011;32:491-509.

25. Barzilai A, Yamamoto K. DNA damage responses to oxidative stress. DNA Repair (Amst). 2004:3:1109-15.

26. Etherton-Beer CD. Vascular cognitive impairment in dementia. Maturitas. 2014;79:220-6.

27. Farkas E, Luiten PG, Bari F. Permanent, bilateral common carotid artery occlusion in the rat: a model for chronic cerebral hypoperfusion-related neurodegenerative diseases. Brain Res Rev. 2007;54:162-80.

28. Otori T, Katsumata T, Muramatsu H, Kashiwagi F, Katayama Y, Terashi A. Longterm measurement of cerebral blood flow and metabolism in a rat chronic hypoperfusion model. Clin Exp Pharmacol Physiol. 2003;30:266-72.

29. Vorhees CV, Williams MT. Value of water mazes for assessing spatial and egocentric learning and memory in rodent basic research and regulatory studies. Neurotoxicol Teratol. 2014;45:75-90.

30. Liu C, Yin H, Gao J, Xu X, Zhang T, Yang Z. Leonurine ameliorates cognitive dysfunction via antagonizing excitotoxic glutamate insults and inhibiting autophagy. Phytomedicine. 2016;23:1638-46.

31. Kadar A, Wittmann G, Liposits Z, Fekete C. Improved method for combination of immunocytochemistry and Nissl staining. J Neurosci Methods. 2009; 184:115-8.

32. Liu H, Zhang J. Cerebral hypoperfusion and cognitive impairment: the pathogenic role of vascular oxidative stress. Int J Neurosci. 2012;122:494-9.

33. Bennett S, Grant MM, Aldred S. Oxidative stress in vascular dementia and Alzheimer's disease: a common pathology. J Alzheimers Dis. 2009;17:245-57.

34. Dubinina EE, Kovrugina SV, Konovalov PV. The factors of oxidative stress in neurodegenerative diseases (vascular dementia, Alzheimer disease). Adv Gerontol. 2007;20:109-13.

35. Kim HA, Miller AA, Drummond GR, Thrift AG, Arumugam TV, Phan TG, et al Vascular cognitive impairment and Alzheimer's disease: role of cerebral hypoperfusion and oxidative stress. Naunyn-Schmiedeberg's Arch Pharmacol. 2012;385:953-9.

36. Lim J, Luderer U. Oxidative damage increases and antioxidant gene expression decreases with aging in the mouse ovary. Biol Reprod. 2011;84 775-82.

37. Mazlumoglu MR, Ozkan O, Alp HH, Ozyildirim E, Bingol F, Yoruk O, et al. Measuring oxidative DNA damage with 8-hydroxy-2'-deoxyguanosine levels in patients with laryngeal cancer. Ann Otol Rhinol Laryngol. 2017;126:103-9.

38. Chien JW, Wang LY, Cheng YS, Tsai YG, Liu CS. Urinary 8-hydroxy-2'-deoxyguanosine (8-oxodG) level can predict acute renal damage in young children with urinary tract infection. Biomarkers. 2014;19:326-31.

39. Mehrdad R, Aghdaei S, Pouryaghoub G. Urinary 8-hydroxy-deoxyguanosine as a biomarker of oxidative DNA damage in employees of subway system. Acta Med Iran. 2015:53:287-92.

40. Ock CY, Kim EH, Choi DJ, Lee HJ, Hahm KB, Chung MH. 8-Hydroxydeoxyguanosine: not mere biomarker for oxidative stress, but remedy for oxidative stressimplicated gastrointestinal diseases. World J Gastroenterol. 2012;18:302-8.

41. Gao X, Lai CQ, Scott T, Shen J, Cai T, Ordovas JM, et al. Urinary 8-hydroxy-2deoxyguanosine and cognitive function in Puerto Rican adults. Am J Epidemiol. 2010;172:271-8.

42. Shi GX, Liu CZ, Wang LP, Guan LP, Li SQ. Biomarkers of oxidative stress in vascular dementia patients. Can J Neurol Sci. 2012;39:65-8.

43. Kroese LJ, Scheffer PG. 8-Hydroxy-2'-deoxyguanosine and cardiovascular disease: a systematic review. Curr Atheroscler Rep. 2014;16:452

44. Luca M, Luca A, Calandra C. The role of oxidative damage in the pathogenesis and progression of Alzheimer's disease and vascular dementia. Oxid Med Cell Longev. 2015;2015:504678.

45. Gackowski D, Rozalski R, Siomek A, Dziaman T, Nicpon K, Klimarczyk M, et al Oxidative stress and oxidative DNA damage is characteristic for mixed Alzheimer disease/vascular dementia. J Neurol Sci. 2008;266:57-62.

46. Hirayama A, Yoh K, Nagase S, Ueda A, Itoh K, Morito N, et al. EPR imaging of reducing activity in Nrf2 transcriptional factor-deficient mice. Free Radic Biol Med. 2003;34:1236-42. 
47. Suzuki T, Yamamoto M. Molecular basis of the Keap1-Nrf2 system. Free Radic Biol Med. 2015;88:93-100.

48. Niture SK, Khatri R, Jaiswal AK. Regulation of Nrf2-an update. Free Radic Biol Med. 2014;66:36-44.
49. Huang Y, Li W, Su ZY, Kong AN. The complexity of the Nrf2 pathway: beyond the antioxidant response. J Nutr Biochem. 2015;26:1401-13.

50. Chapple SJ, Siow RC, Mann GE. Crosstalk between Nrf2 and the proteasome: therapeutic potential of Nrf2 inducers in vascular disease and aging. Int J Biochem Cell Biol. 2012;44:1315-20. 